\title{
Groundwater Maps of the Hanford Site, June 1993
}

FEB $28: 154$

OSTI

Prepared for the U.S. Department of Energy Office of Environmental Restoration and Waste Management

\section{(20) Westinghouse}

Hanford Company Richland, Washington

Hanford Operations and Engineering Contractor for the

U.S. Department of Energy under Contract DE-AC06-87RL10930

Approved for Public Release 
LEGAL DISCLAIMER

This report was prepared as an account of work sponsored by an agency of the United States Government. Neither the United States Government nor any agency thereof, nor any of their employees, nor any of their contractors, subcontractors or their employees, makes any warranty, express or implied, or assumes any legal liability or responsibility for the accuracy, completeness, or any third party's use or the results of such use of any information, apparafus, product, or process disclosed, or represents that its use would not infringe privately owned rights. Reference herein to any specific commercial product, process, or service by trade name. trademark, manufacturer, or otherwise, does not necessarily constitute or imply its endorsement, recommendation, or favoring by the United States Government or any agency thereof or its contractors or subcontractors. The views and opinions of authors expressed herein do not necessarily state or reflect these of the United States Government or any agency thereot.

This report has been reproduced from the best available copy. Available in paper copy and microfiche.

Available to the U.S. Department of Energy and its contractors from

Office of Scientific and Technical Information

P.0. Box 62

Oak Ridge, TN 37831

(615) 576-8401

Available to the public from the U.S. Department of Commerce National Technical Information Service

5285 Port Royal Road

Springfield, VA 22161

(703) 487.4650

Printed in the Unilod Statos of Amorica

DISCLM-1.CHP (1-81) 


\section{Groundwater Maps of the Hanford Site, June 1993}

G. L. Kasza

M. J. Hartman

W. A. Jordan

D. C. Weekes

Date Published

February 1994

Prepared for the U.S. Department of Energy Office of Environmental Restoration and Waste Management

\section{(2.) Westinghouse P.O. Box 1970 \\ (W) Hanford Company Richland, Washington 99352 \\ Haniord Operations and Engineering Contractor for the \\ U.S. Department of Energy under Contract DE-AC06-87RL10930}




\section{CONTENTS}

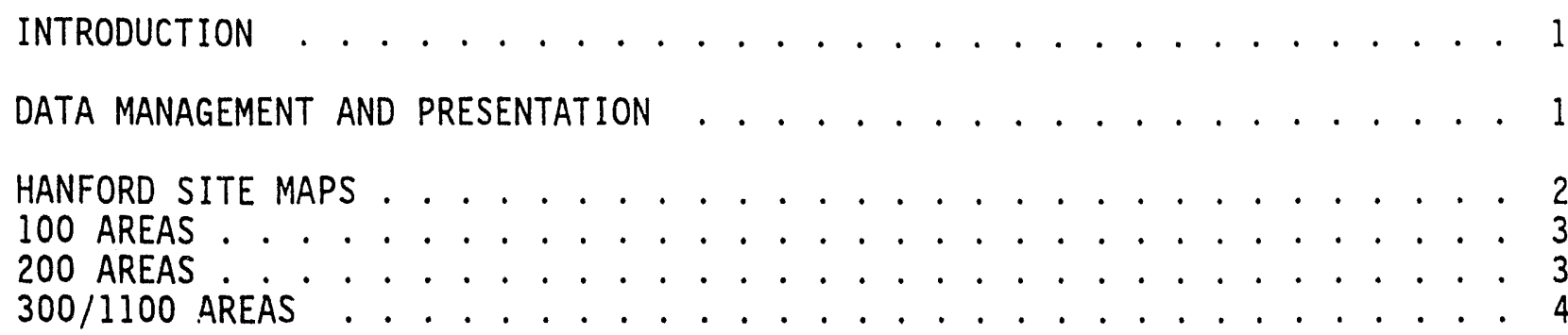

REFERENCES . . . . . . . . . . . . . . . . . . . 5

\section{FIGURES :}

1 Hanford Site Location Map . . . . . . . . . . . . . . . . . . . 7

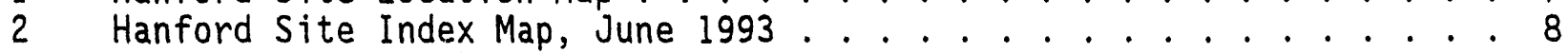

3 Hanford Site Depth-to-Water Map, June 1993 . . . . . . . . . . . . . 9

4 Hanford Site Water Table Map, June 1993 . . . . . . . . . . . . . . . 10

5100 Areas Index Map, June 1993 . . . . . . . . . . . . . . . . . . . . 11

6100 Areas Depth-to-Water Map, June 1993................. 12

7100 Areas Water Table Map, June 1993 . . . . . . . . . . . . . . . . 13

8200 Areas Index Map, June 1993 . . . . . . . . . . . . . . . . . . . 14

9200 Areas Depth-to-Water Map, June 1993 . . . . . . . . . . . . . . . 15

10200 Areas Water Table Elevation, June 1993 . . . . . . . . . . . . . 16

$11300 / 1100$ Areas Index Map, June 1993 . . . . . . . . . . . . . . . . . 17

$12300 / 1100$ Areas Depth-to-Water Map, June 1993 . . . . . . . . . . . . 18

$13300 / 1100$ Areas Water Table Map, June 1993 . . . . . . . . . . . . . . 19

APPENDIX A - WATER LEVEL MEASUREMENT DATA . . . . . . . . . . . . . . . . A-1 
WHC-EP-0394-7

\section{INTRODUCTION}

The Groundwater Maps of the Hanford Site, June 1993 is an update of the series of reports that document the configuration of the uppermost unconfined aquifer beneath the Hanford Site (Figure 1). This report series presents the semiannual water level measurements taken at site groundwater monitoring wells each June and December and the groundwater maps derived from these

measurements. These reports document the changes in the groundwater level at Hanford as the site has transitioned from nuclear material production to environmental restoration and remediation. In addition, these reports provide water level data to support the various site characterization and groundwater monitoring programs currently in progress on the Hanford Site. Groundwater Maps of the Hanford Site are prepared for the U.S. Department of Energy, Office of Environmental Restoration and Waste Management, by the Hanford Site Operations and Engineering Contractor, Westinghouse Hanford Company (WHC). This document fulfills reporting requirements specified in WHC (1993), Section 8.0 "Water Quality" and also described in the Environmental Monitoring Plan for the Hanford Site (DOE-RL 1991).

Maps depicting the water table beneath the Hanford Site south of the Columbia River are presented in this report. Appendix A 1 ists the we 11 identification number, depth to water, casing elevation and the water level elevation for each well measured during June 1993. A summary discussion of the data is included with a well index map, the depth to water map and the contoured map of the water table surface for the Hanford Site and each of the three operational areas (the 100, 200, and 300-1100 Areas). For clarity, the locating prefixes have been omitted from all well numbers shown on the maps. Wells in the 100 Areas have the prefix 199; those in the 200 Areas have the prefix 299; wells in the 300 Area have a 399 prefix; those in the 1100 Area have a 1199 prefix and the wells outside these areas have the prefix 699 . An explanation of the Hanford well numbering system and a listing of the survey coordinates for each well drilled prior to 1993 is listed in Chamness and Merz (1993).

The National Geodetic Vertical Datum of 1929 is used as the vertical datum and the Hanford Plant Coordinates are used for horizontal location of the wells. Both datums are measured in feet.

This report and groundwater maps were prepared by staff members of the WHC Geohydrologic Engineering Function, Environmental Division. Water level data from the previous report period (December 1992) were reported in Kasza et al. (1993).

\section{DATA MANAGEMENT AND PRESENTATION}

During June 1993, the depth to the groundwater was measured in 751 Hanford Site wells by personnel from Subsurface Investigative Support Function under the direction of the Geohydrologic Engineering Function project leaders. The procedure for measuring the depth to water and recording the data is contained in environmental investigations instructions (EII) 10.2 
WHC-EP-0394-7

(WHC 1988). Field data were collected using the Groundwater Monitoring System, which employs a barcode interface to enter readings that have been taken manually with a steel tape from the reference point at the top of the well casing. The barcode hand-held computer downloads the field data to a supervisory workstation that stores all groundwater level measurements. These data are transferred to the Hanford Environmental Information System (HEIS) data base and were later downloaded to a Paradox (a trademark of Borland International) application program for use by the Geohydrologic Engineering function hydrologists.

The Paradox application was used to classify, provide coordinates for, and format data points used in creating contours of the water table across the Hanford Site. Data were averaged for wells with multiple measurements in June. The Paradox application was also used to generate the data tables presented in Appendix $A$.

Contours generated from groundwater data are displayed on the water table elevation maps and were created within Earth Vision (a trademark of Dynamic Graphics, Incorporated. Earth Vision is software that calculates a data grid and generates contour lines when given a set of data point locations and values. A 2-D minimum tension gridding algorithm was used to interpolate grid values. Generated contour lines were then imported into an AutoCad (a trademark of Autodesk, Incorporated) environment to be overlaid onto existing base maps.

Hydrologists familiar with regional and local groundwater properties reviewed the maps to evaluate data interpolations made by the Earth Vision software. A representative set of wells was illustrated in areas where there are heavy concentrations of wells (e.g., 100-N and 100-H Areas). Hydrographs were plotted for wells with anomalous head values. If the measurement was an outlier, the data point was not included in constructing the maps and its questionable status was noted in Appendix A. Map modifications and corrections were made in Autocad and the final maps were generated.

\section{HANFORD SITE MAPS}

The Hanford Site maps depict the configuration of the surface of the unconfined aquifer beneath that portion of the Hanford Site located south and west of the Columbia River. The site maps include:

- Figure 2, Index Map of the Hanford Site

- Figure 3, Site Depth-to-Water Map

- Figure 4, Hanford Site Water Table Map.

The Hanford Site water table map is a composite of the detailed maps of the operating areas and data from the groundwater monitoring wells located outside the operating areas. The borders of the operating areas maps are represented accurately on the site maps for ease in comparing the maps. 
The "areas where the basalt surface is generally above the water table" reflect the structural contour of the top of basalt according to connelly et a1. (1992a and 1992b).

\section{AREAS MAPS}

For the purposes of this report, the 100 Areas comprise the various 100 Area inactive reactor facilities and the surrounding land south of the Columbia River and north of Gable Mountain and Gable Butte. Maps for this area include:

- Figure 5, Index Map of the 100 Areas Groundwater Monitoring Wells

- Figure 6, 100 Areas Depth-to-Water Map

- Figure 7, 100 Areas Water Table Map.

The maps are based on June 1993 field measurements from those wells that are located north of Hanford Site coordinate N56,000 and completed in the unconsolidated sediments. Wells used are open to the aquifer within $100 \mathrm{ft}$ of the average water table since vertical gradients are not significant on the scale of the map.

River stage recorders are located in the 100-B/C, 100-H, and 100-F Areas. River stage is measured hourly with pressure transducers. The averages of the June values are plotted on Figure 7 and were used in contouring the water table map.

Throughout most of the map area, groundwater flows from the unconfined aquifer into the Columbia River. West of the 100-B/C Area, however, water is believed to flow from the river into the aquifer.

The high water levels north of Gable Mountain are consistent with past measurements. Driller's logs indicate the presence of fine-grained sediments in this area. The high water levels may be remnants of artificial recharge from pre-Hanford irrigation, or may represent perched groundwater. The steep groundwater gradient in the southeastern portion of the map area is believed to result from the influence of the fine-grained sediments.

Water level data used to construct the maps are listed in Appendix A. The $N$ 65,000 Separations Area border line is included on the maps of the 100 and 200 Areas for convenient reference.

\section{AREAS MAPS}

These maps encompass the 200 East and 200 west Areas and the surrounding vicinity on the Hanford Site, which was once referred to as the Separations Area. The semiannual measurement of water levels in the 200 Areas was performed on the more than 180 selected wells that comprise the Operational Groundwater Monitoring Network (Serkowski and Jordan, 1989). The Operational 
Groundwater Monitoring Network provides water level measurements for the determination of the water table configuration and for the water quality sampling of the groundwater beneath and surrounding the chemical processing and the waste management facilities in the 200 Areas. Water level data gathered for the ongoing Resource Conservation and Recovery Act groundwater monitoring program, the Comprehensive Environmental Response, Compensation and Liability Act site characterization activities, and from outlying wells are also included on the maps for a more complete representation of the water table. The wells selected were reviewed to assure that they were completed in the unconsolidated sediments and screened or perforated in the upper portion of the unconfined aquifer.

Groundwater beneath the 200 Areas flows northward through the gap between the Gable Butte-Gable Mountain basalt highs or eastward toward the Columbia River (Figure 4). The major influences on water table elevations beneath the 200 Areas are waste water currently discharged to cribs, trenches and the B-Pond system, and the pre-existing water table mounds beneath the Areas. The water table had been artificially elevated since the start of the nuclear processing operations due to the large volumes of water disposed to the soil but has been falling in recent years because of the large-scale reduction in process water usage after the end of nuclear material production. Kasza et a1. (1993) presents a map depicting the change in water table elevation between December 1988 and December 1992.

The 200 Areas set of maps includes:

- Figure 8, 200 Areas Monitoring Well Index Map

- Figure 9, 200 Areas Depth-to-Water Map

- Figure 10, 200 Areas Water Table Map.

Representative wells were selected to portray the water level data on the maps where the concentration of monitoring wells would produce a crowded illustration.

An area of conflicting data is noted on Figure 10. Within this area, use of the measured depth-to-water produces an improbable calculated water table elevation. Reference point elevation errors are suspected as the cause. Information for these wells (contained in Appendix A) are noted as being of suspect quality $(Q)$.

\section{$300 / 1100$ AREAS MAPS}

These maps present the June 1993 water table measurements taken at the 300 Area, the adjacent 1100 Area, and the Horn Rapids Landfill site.

Measurements were made in wells that were screened or perforated in the upper part of the unconfined aquifer except in a few cases where the top of the screen was slightly below the top of the unconfined aquifer. In the map area, the top of the unconfined aquifer is very close to the Hanford formation/ Ringold Formation contact. Generally, the unconfined aquifer west of an imaginary north-south line near the west boundary of the 300 Area is within the Ringold Formation. East of the line, the top of unconfined aquifer is 
WHC-EP-0394-7

within the Hanford formation. The maps for this part of the Hanford Site include:

- Figure 11, 300/1100 Areas Index Map

- Figure 12, 300/1100 Areas Depth-to-Water Map

- Figure 13, 300/1100 Areas Water Table Map.

The major influences on water table elevations in the map area are river level fluctuations, waste water discharged to the 300 Area process trenches and sanitary system, and river water recharged into the city of Richland well field near the 1100 Area. Water table fluctuations are also related to irrigation of farm lands and recharge from the Yakima River, both of which are located west of the map area. The groundwater mound centered on well 699-S40-E14 is located beneath the city of Richland well field and is the result of groundwater recharge.

A pressure transducer, located at station SWS-1, monitors the Columbia River stage at the 300 Area. River stage readings are taken every hour and stored in a data logger, which is then downloaded to a computer data base. The river stage measurement for the day and time that the 300 Area wells were measured is presented on the maps since the water level in the unconfined aquifer near the Columbia River responds rapidly to variations in the river stage.

\section{REFERENCES}

Chamness, M. A. and J. K. Merz, 1993, Hanford Wel7s, PNL-8800, Pacific Northwest Laboratory, Richland, Washington.

Connelly, M. P., B. H. Ford, and J. V. Borghese, 1992a, Hydrogeologic Model for the 200 West Groundwater Aggregate Area, WHC-SD-EN-TI-014, Westinghouse Hanford Company, Richland, Washington.

Connelly, M. P., B. H. Ford, J. W. Lindberg, S. J. Trent, C. D. Delaney, and J. V. Borghese, 1992b, Hydrogeologic Model for the 200 East Groundwater Aggregate Area, WHC-SD-EN-TI-019, Westinghouse Hanford Company, Richland, Washington.

DOE-RL, 1991, Environmental Monitoring P7an, DOE/RL 91-50, U.S. Department of Energy, Richland Field Office, Richland, Washington.

Kasza, G. L., M. J. Hartman, F. N. Hodges, and D. C. Weskes, 1993, Groundwater Maps of the Hanford Site--December 1992, WHC-E.P-0394-6, Westinghouse Hanford Company, Richland, Washington.

Serkowski, J. A. and W. A. Jordan, 1989, Operational Groundwater Monitoring at the Hanford Site--1988, WHC-EP-0260, Westinghouse Hanford Company, Richland, Washington.

WHC, 1988, Environmental Investigations and Site Characterization Manual, WHC-CM-7-7, Westinghouse Hanford Company, Richland, Washington. 


\section{WHC-EP-0394-7}

WHC, 1993, Environmental Compliance, WHC-CM-7-5, Westinghouse Hanford Company, Richl and, Washington. 


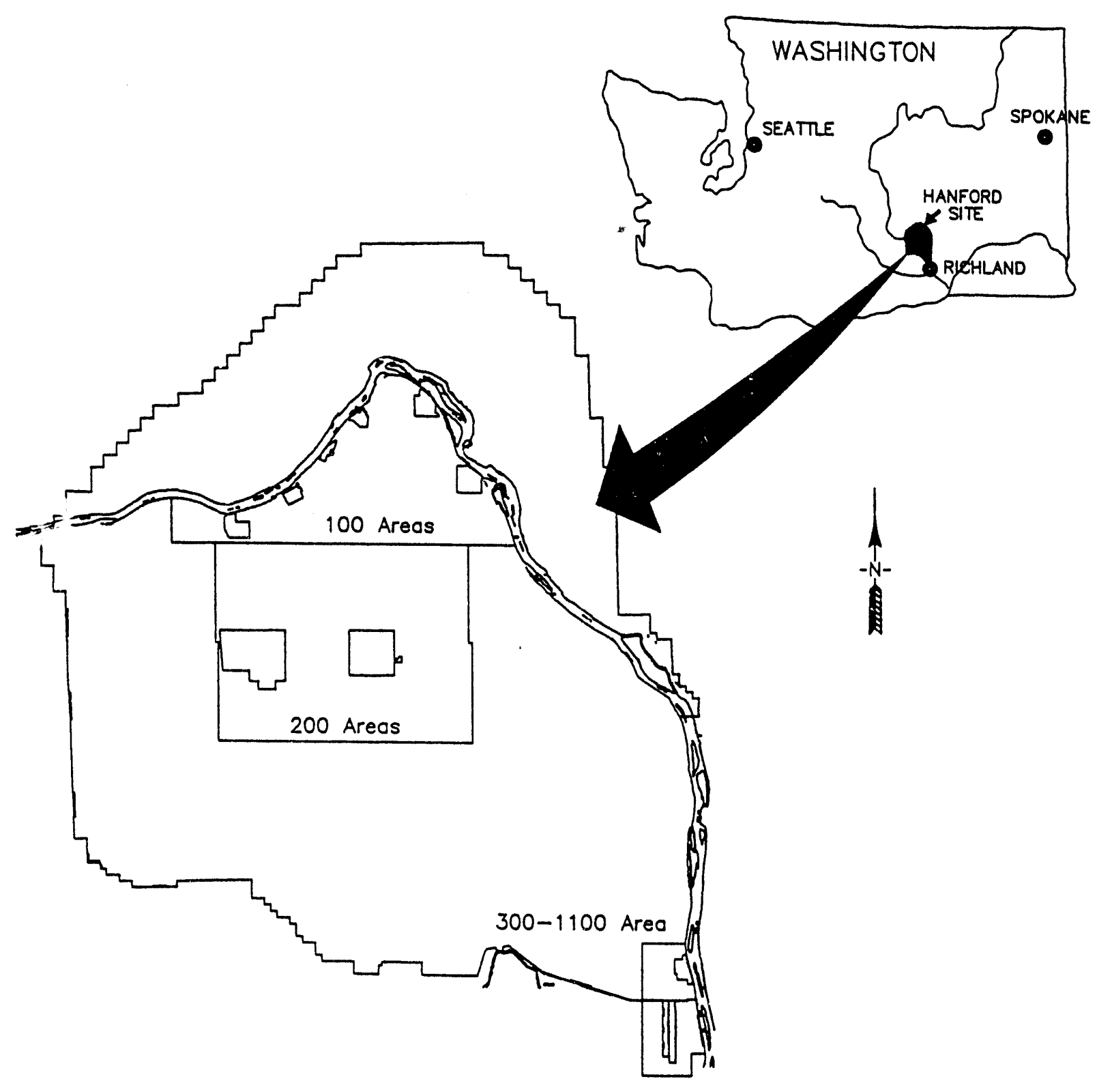

Figure 1. Hanford Site Location Map. 


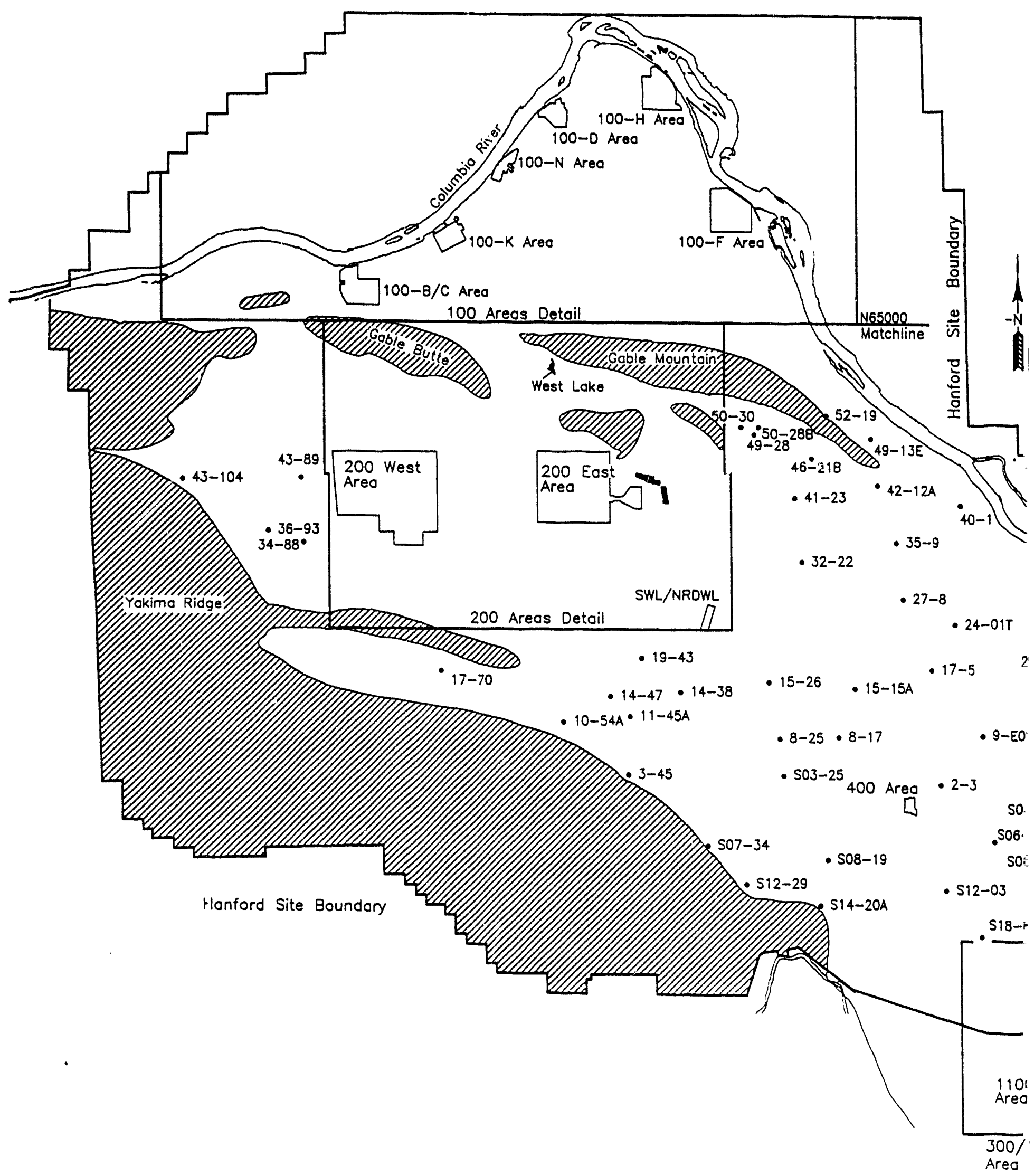


WHC-EP-0394-7

\title{
Hanford Site Index Map June 1993
}

\author{
32-22 Identification of monitoring well used \\ to prepare this map. All wells on this \\ map are prefixed by 699-. \\ (1) Ponds \\ Areas where the basalt surface is \\ generally above the water table. \\ The Hanford site index map has been \\ prepared by the Geohydrologic Engineering \\ Function, Westinghouse Hanford Company. \\ Note: To convert to metric, multiply \\ elevation ( $\mathrm{ft}$ ) by 0.3048 to obtain \\ elevation (m).
}

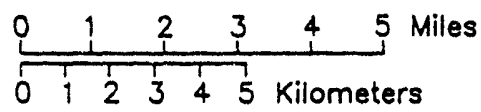

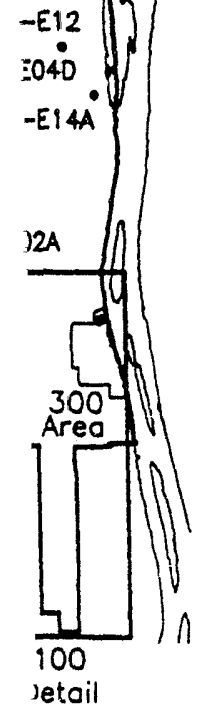

Figure 2

WAJ/610XJN93 


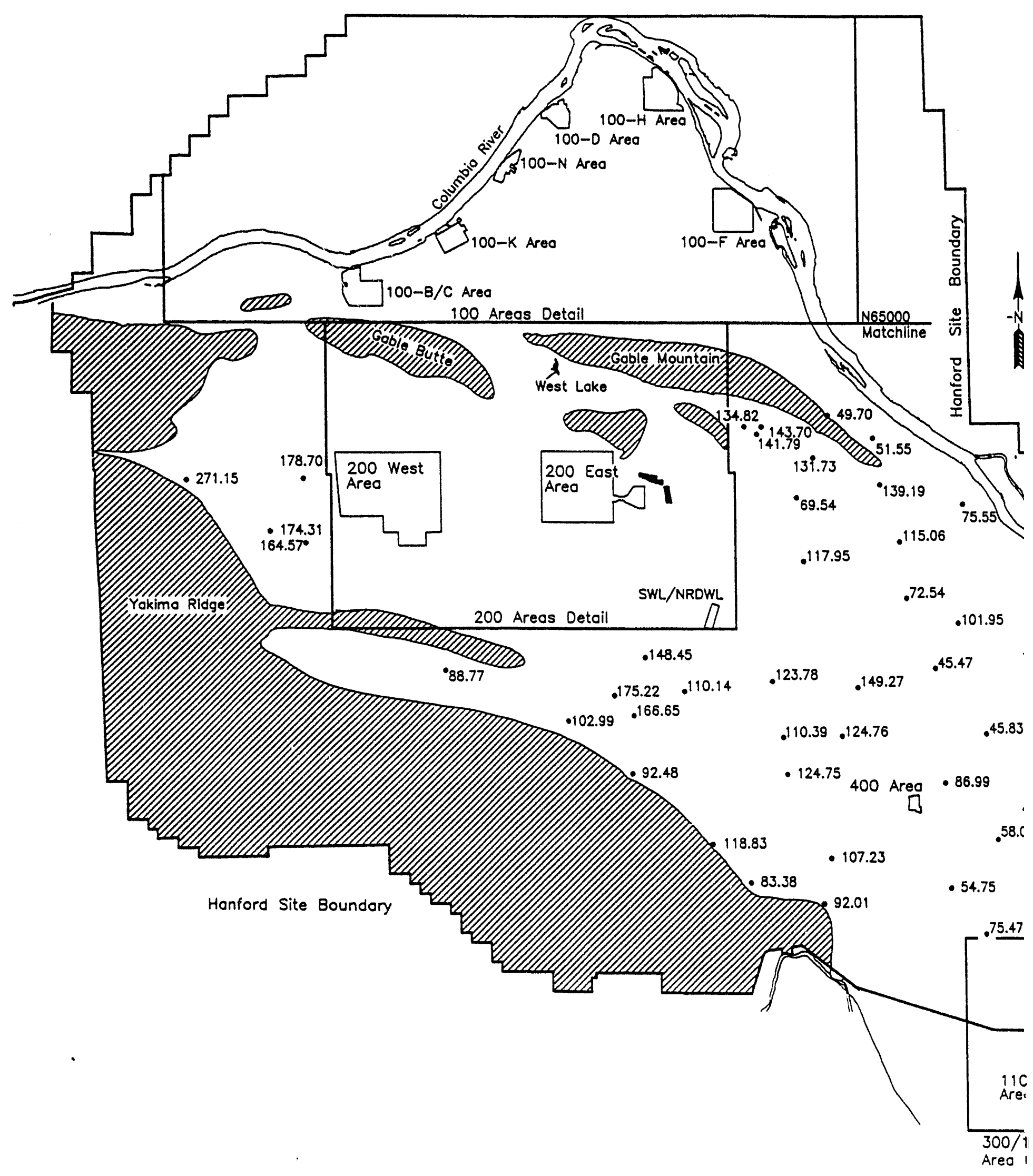


WHC-EP-0394-7

\section{Hanford Site Depth-to-Water Map June 1993}

36.77 Depth to water in feet, as

- measured from well reference mark (generally top of casing) to groundwater surfoce

Ponds

Areas where the bosalt surface is generally above the water table

The Hanford site depth-to-water map has been prepared by the Geohydrologic Engineering Function, Westinghouse Honford Company.

Note: To convert to metric, multiply elevation ( $\mathrm{ft}$ ) by 0.3048 to obtain elevation $(m)$.

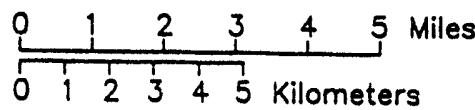




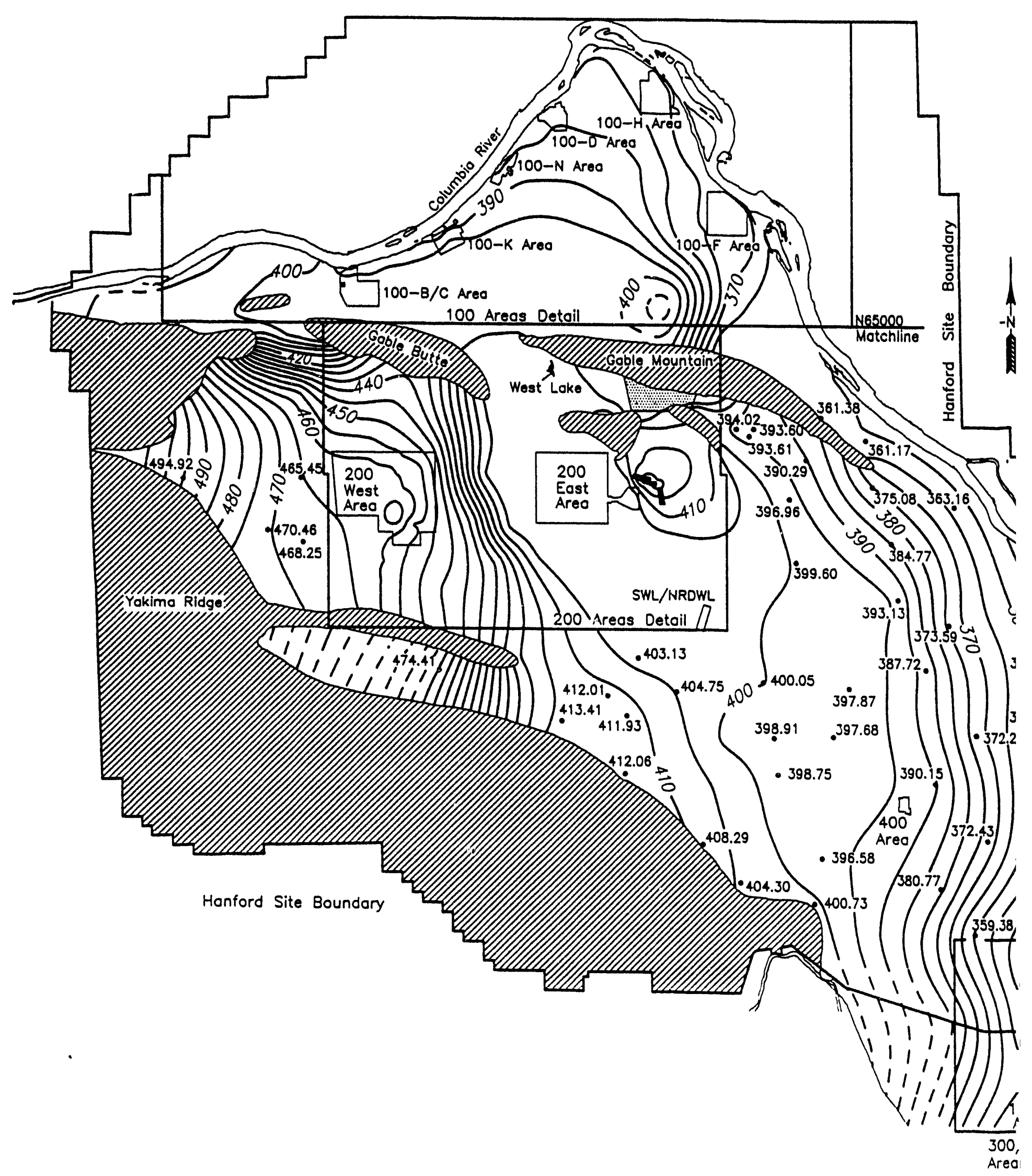




\title{
Hanford Site Water Table Map June 1993
}




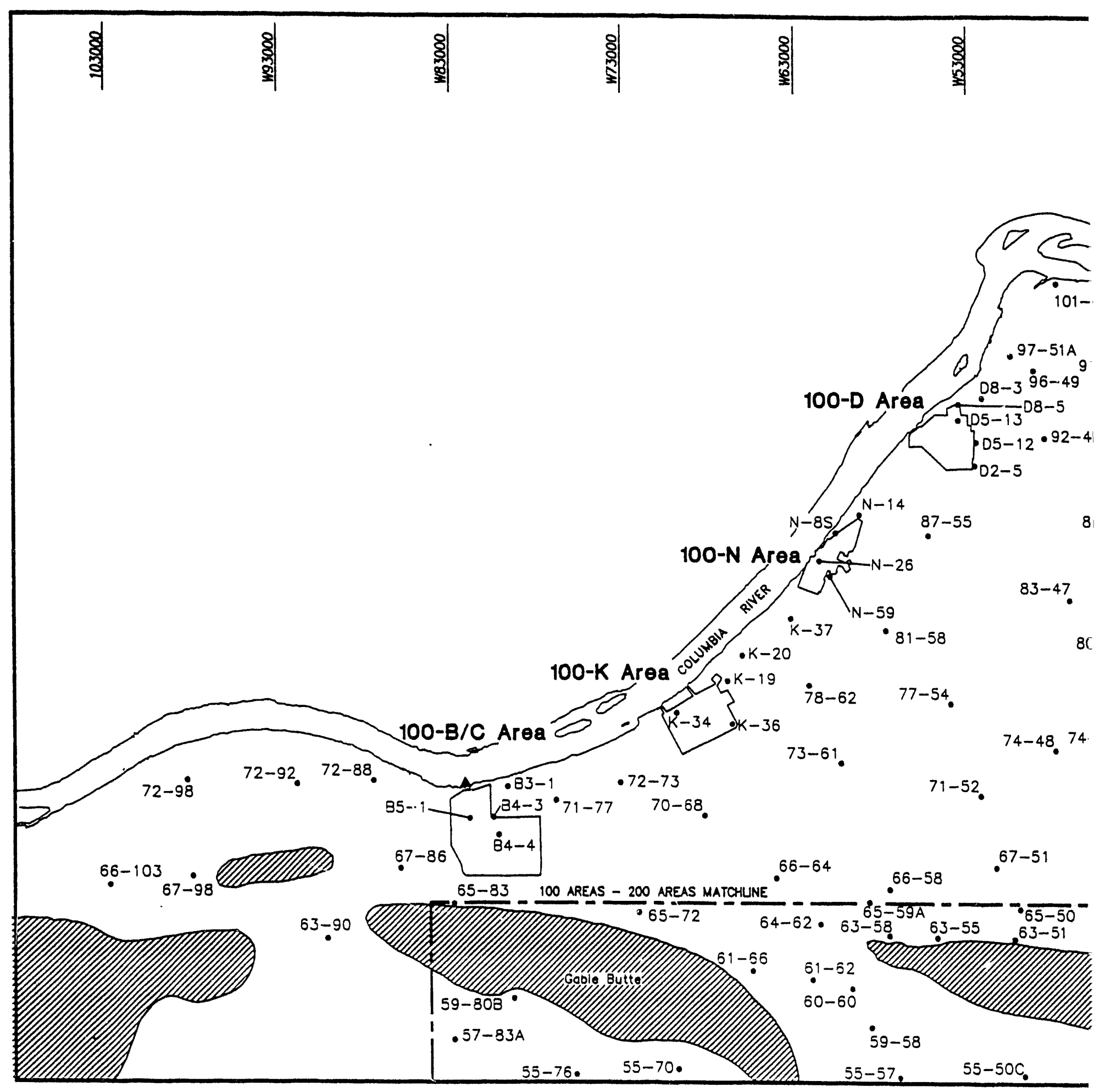




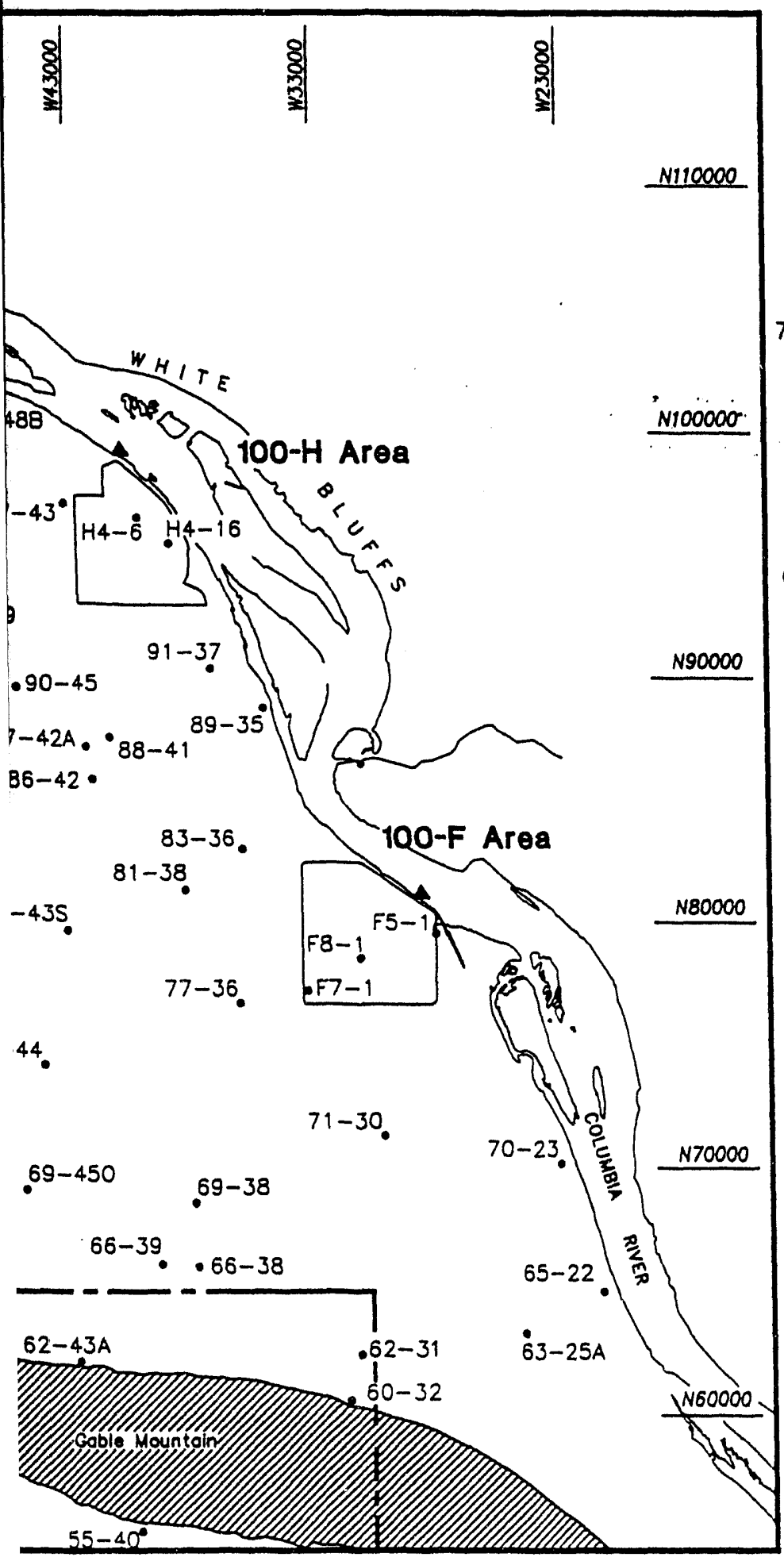

\section{Areas Index Map June 1993}

77-40 Identification of Groundwater Monitoring Wells Used to Prepore this Map. Well numbers beginning with a letter are $\therefore$ prefixed with a 199- and beginning with a number are prefixed with 699-

- River stage recorder

AlII Areas where the basalt surface is generally above the water table

The 100 Areas Index Map has been prepared by the Geohydrologic Engineering Function, Westinghouse Hanford Company.

Note: To convert to metric, multiply elevation ( $\mathrm{ft}$ ) by 0.3048 to obtain elevation $(m)$.

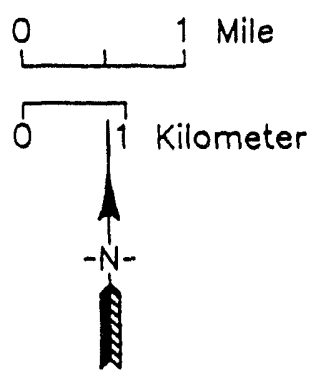

Figure 5

WAJ/110XJN93 


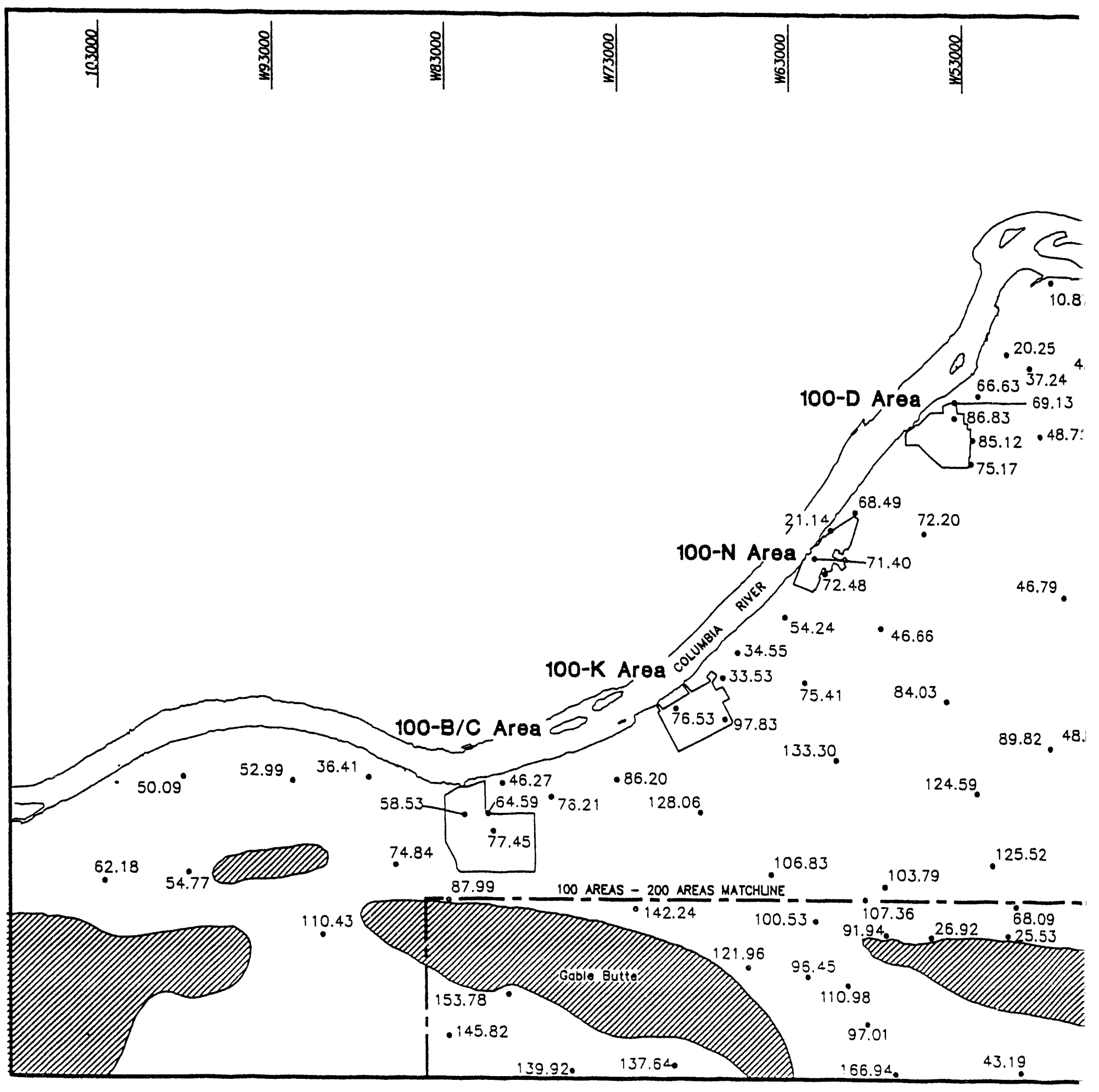


WHC-EP-0394-7

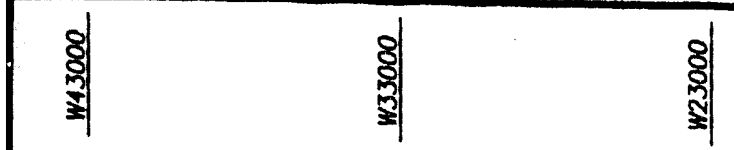

$N 110000$

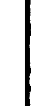

3.<smiles>CC#[14CH]</smiles>

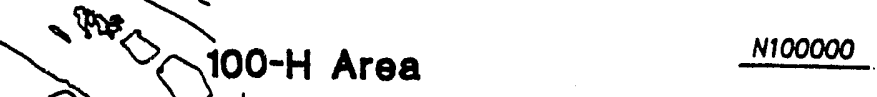

.37 .68

$32.71 \cdot 33.90$

25.41 •

41.03

27.41 .

4.93

36.53 .
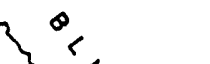

1100000

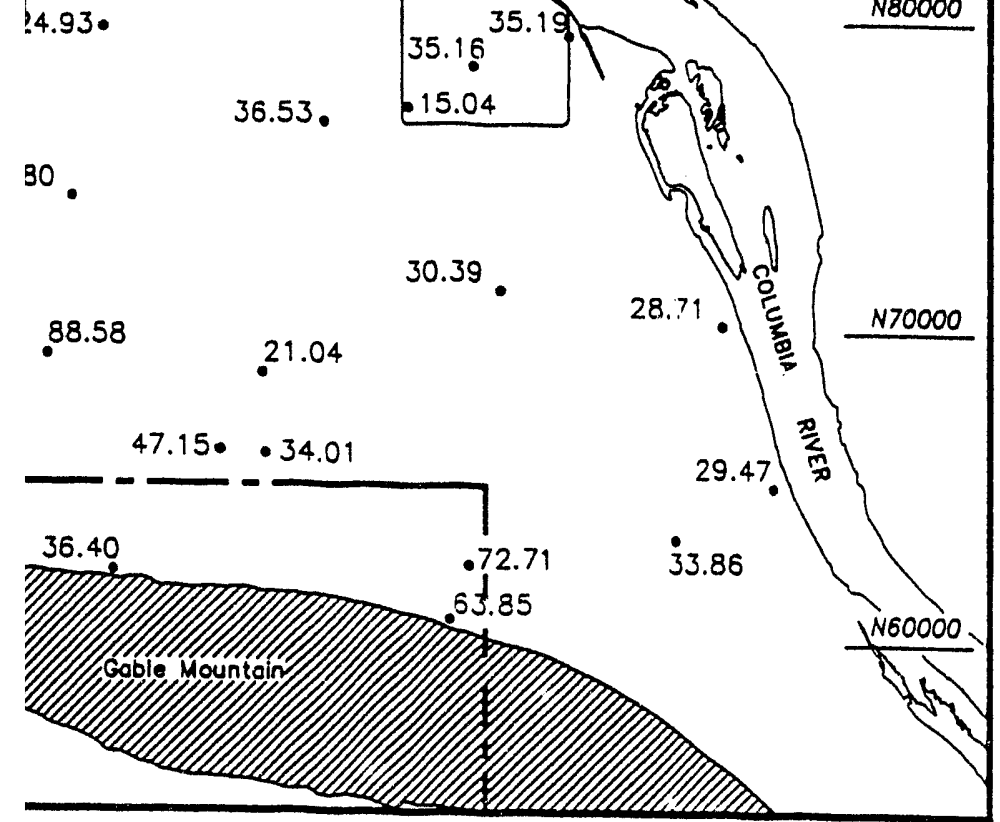

WAJ/10TWJN93

\section{Areas Depth-to-Water Map June 1993}

32.54 Depth to water, as measured from the well reference mark (generally top of cosing) to groundwater surfoce.

שIII

Areas where the bosalt surface is generally above the water table

The 100 Areas depth-to-water map has been prepored by the Geohydrologic Engineering Function, Westinghouse Hanford Company.

Note: To convert to metric, multiply elevation ( $\mathrm{ft}$ ) by 0.3048 to obtain elevation $(\mathrm{m})$.

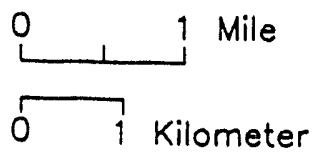

Figure 6 


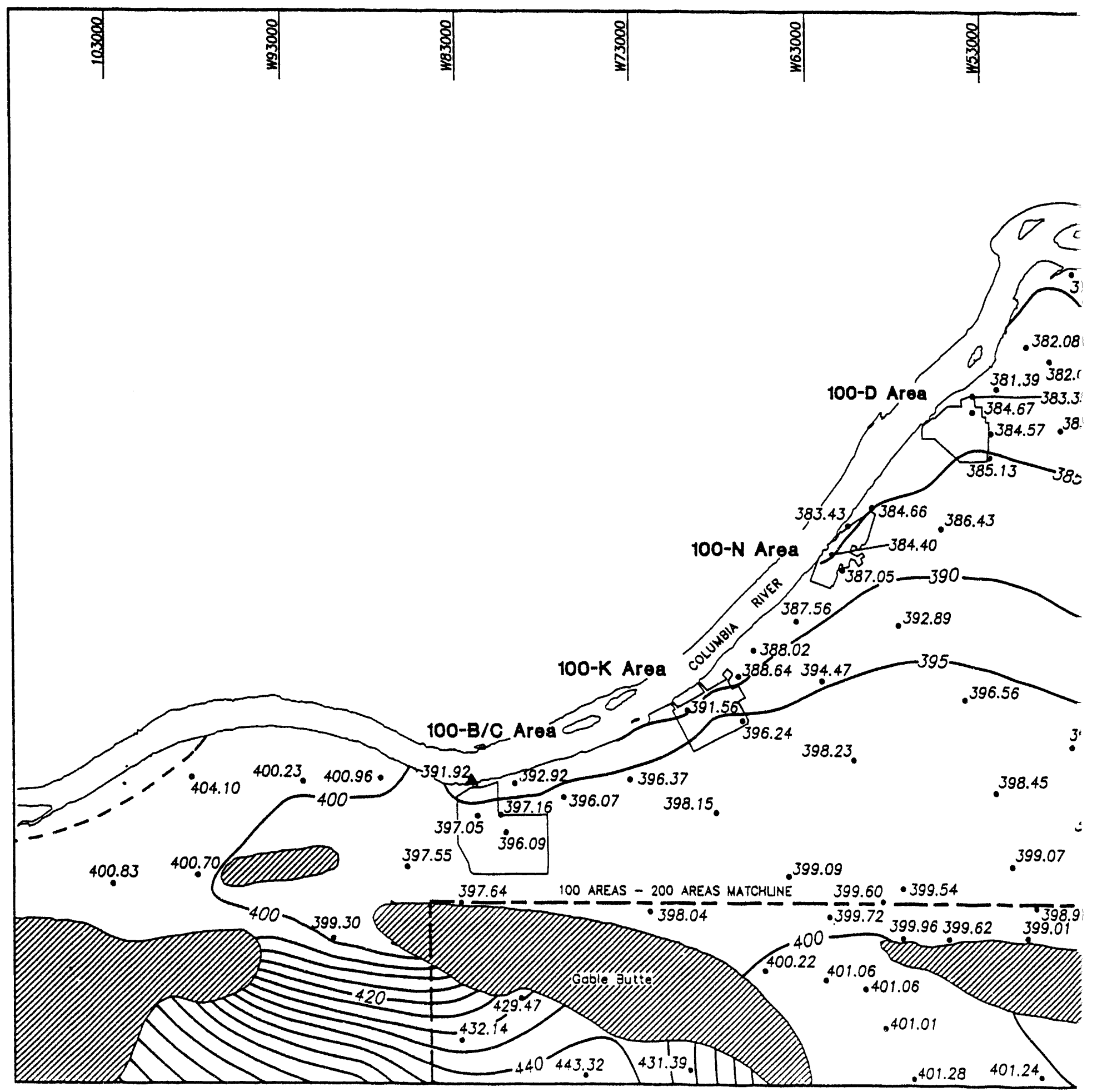


WHC-EP-0394-7

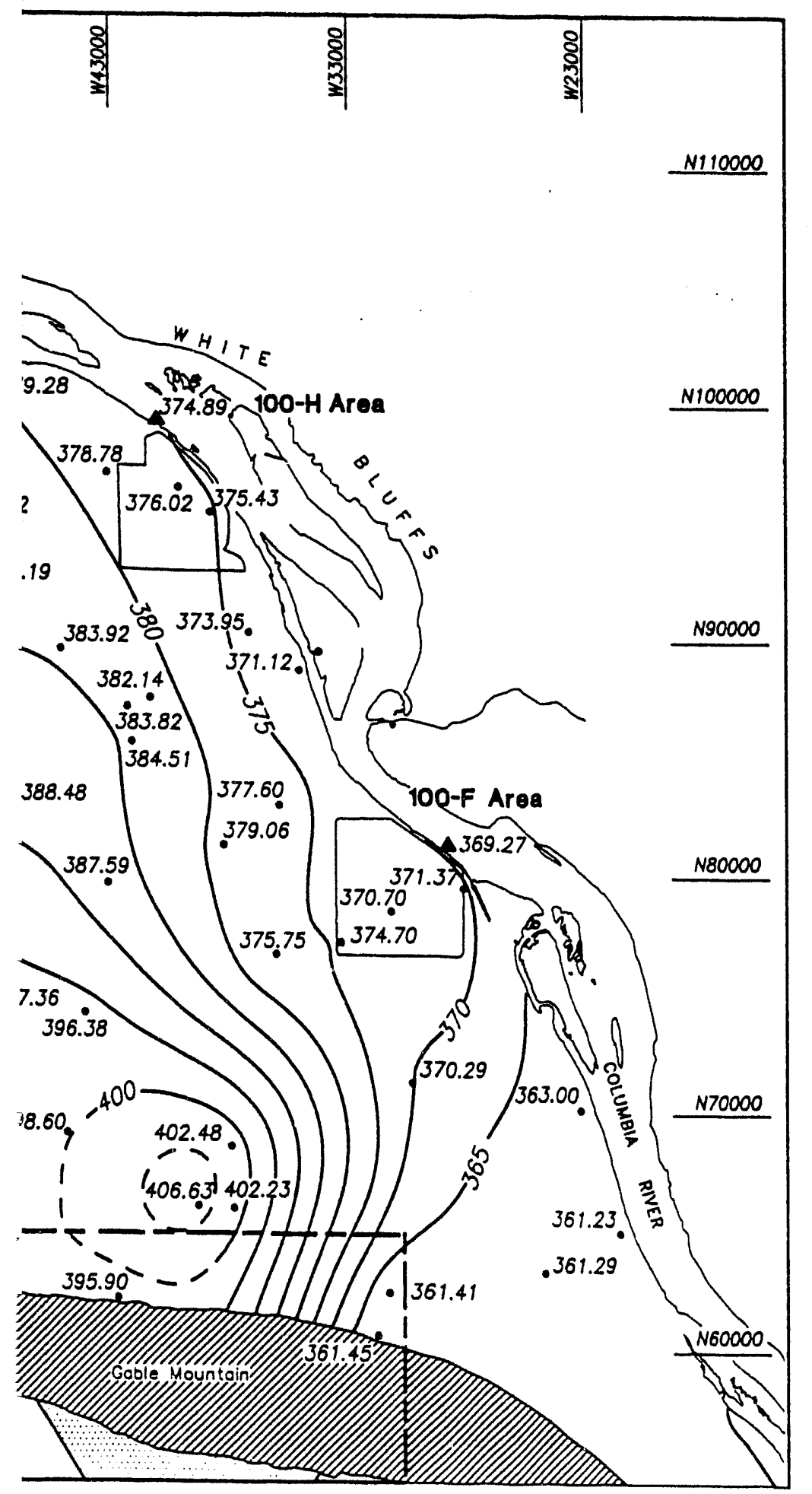

WAJ/ICONJN93

\section{Areas Water Table Map June 1993}

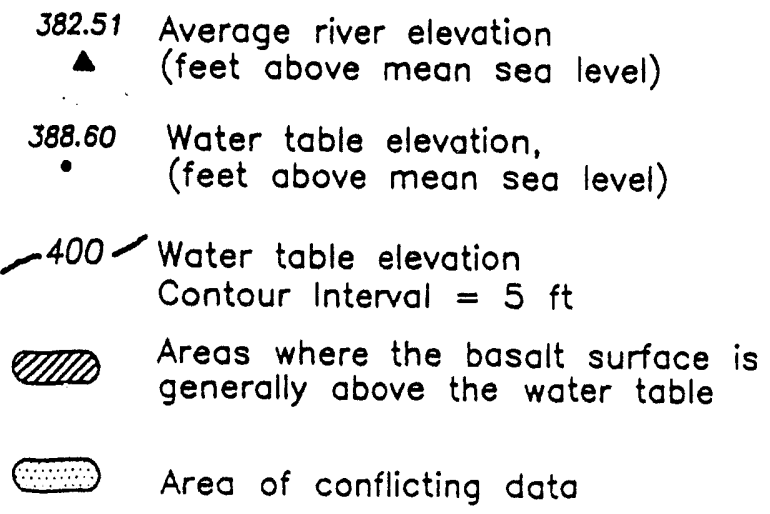

382.51 Average river elevation

- (feet above mean sea level)

388.60 Water table elevation, (feet above mean sea level)

-400 Water table elevation Contour Interval $=5 \mathrm{ft}$

AIID Areas where the basolt surface is generally above the water table

Area of conflicting dato

The 100 Areas water toble map has been prepared by the Geohydrologic Engineering Function. Westinghouse Hanford Company.

Note: To convert to metric, multiply elevation ( $\mathrm{ft}$ ) by 0.3048 to obtain elevation ( $m$ ).

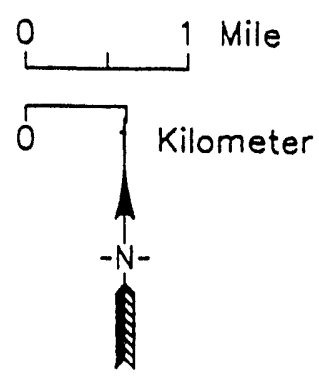




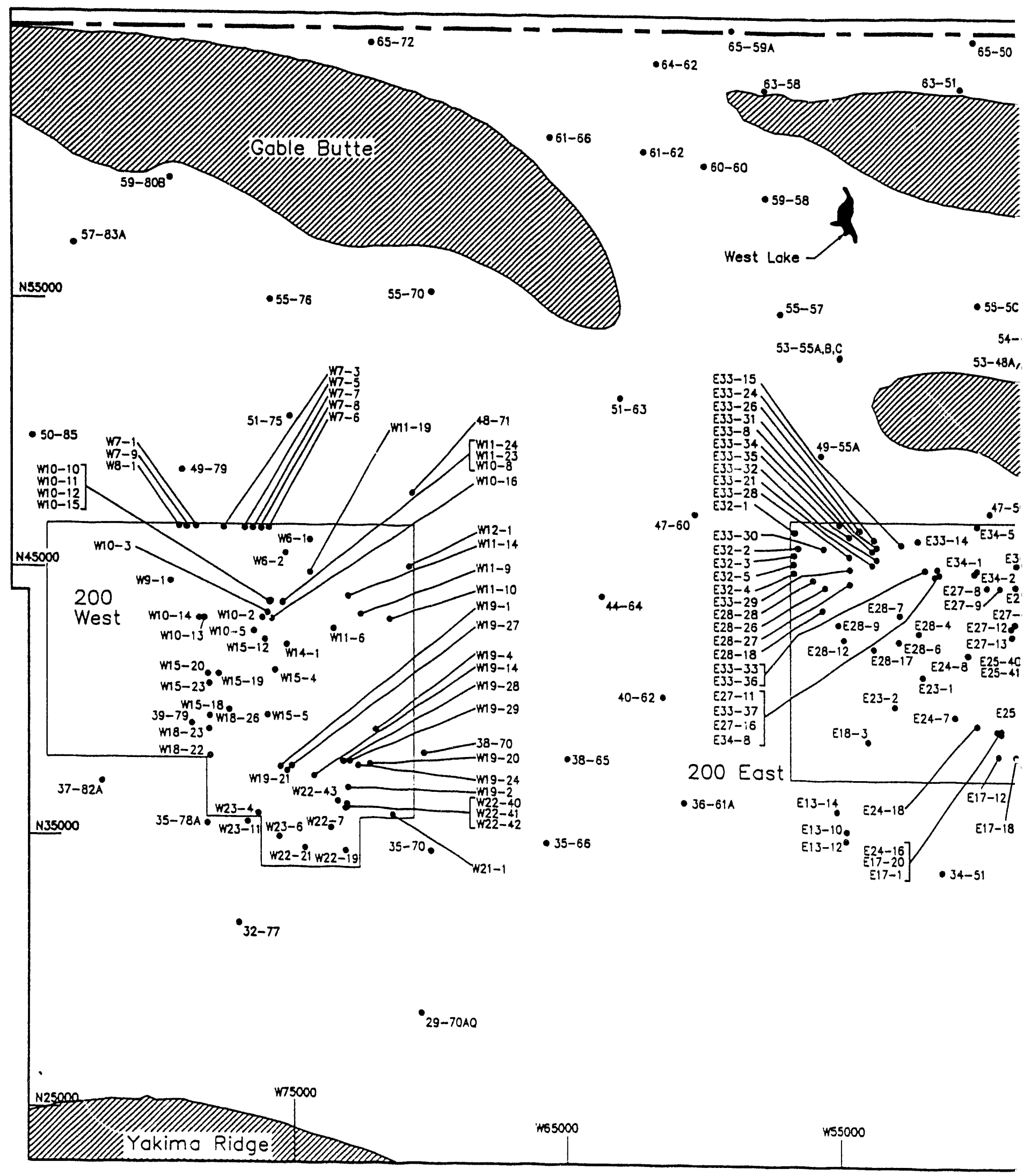




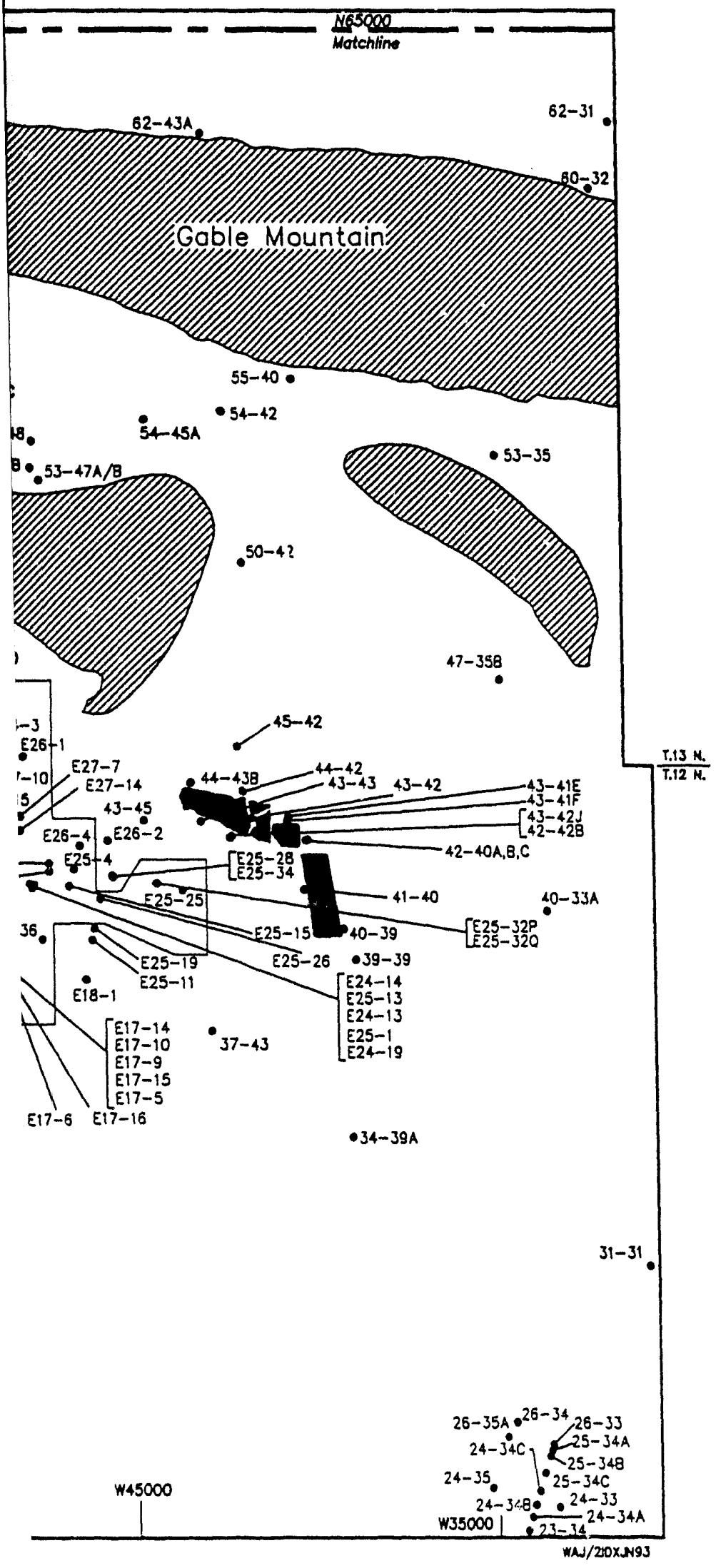

\section{Areas \\ Index Map June 1992}

W22-26 Identification of monitoring well used to prepare map. Well Numbers starting with $E$ or $W$ ore prefixed by $299-$ and all others are prefixed by 699-.

$\longrightarrow$ Ponds

DIII Areas where the bosalt surfoce is generally above the water toble

The 200 Areas index map has been prepared by the Geohydrologic Engineering Function, Westinghouse Hanford Company.

Note: To convert to metric, multiply elevation (ft) by 0.3048 to obtain elevation $(\mathrm{m})$.

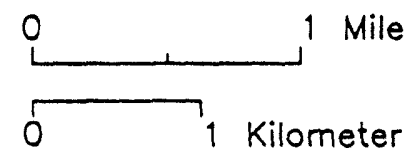<smiles>C=C[N+]1=C(C)CC1</smiles> 


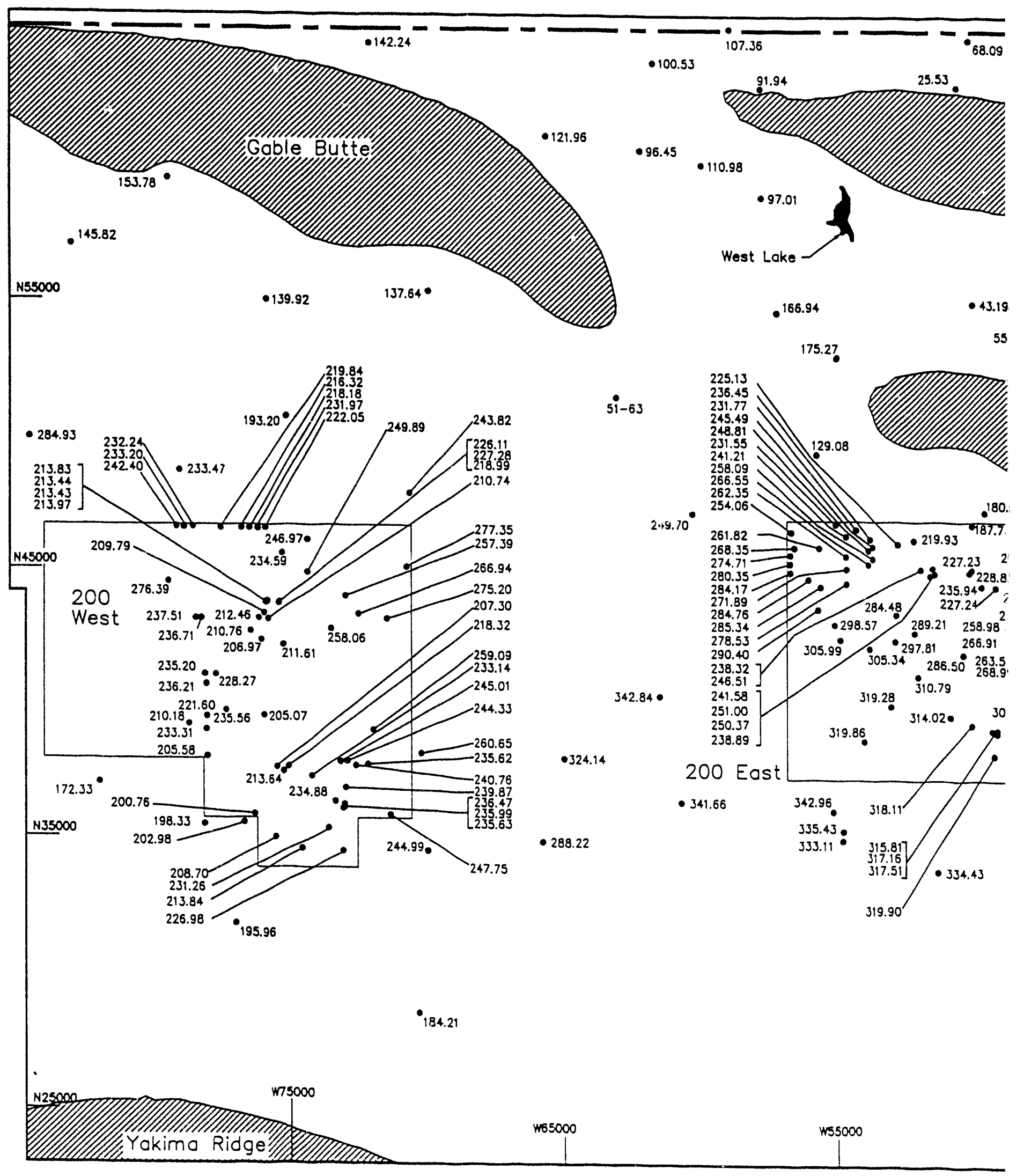




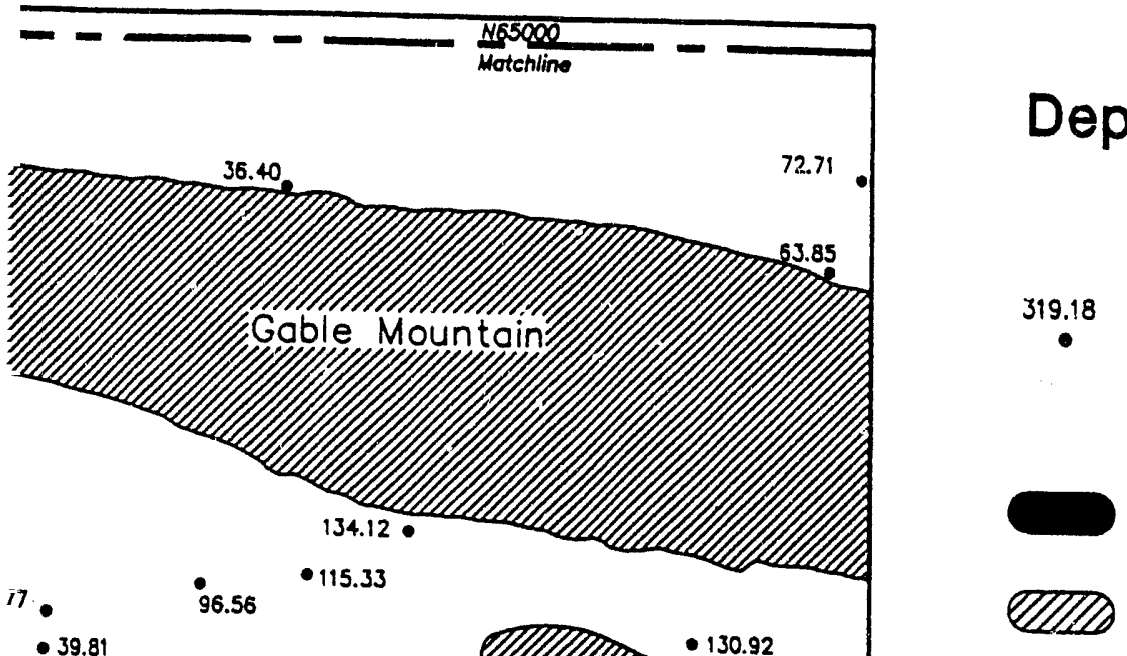

200 Areas Depth-to-Water Map June 1993

19.18 Depth to water in feet, os meosured from well reference mark (generally top of casing) to groundwater surface.

\section{Ponds}

Areas where the bosalt surfoce is generally above the water table

The 200 Areas depth-to-water map has been prepared by the Geohydrologic Engineering Function, Westinghouse Hanford Company.

Note: To convert to metric, multiply elevotion ( $\mathrm{ft}$ ) by 0.3048 to obtain elevation ( $m$ ).
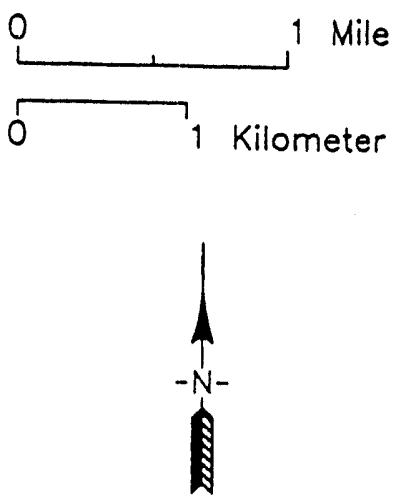

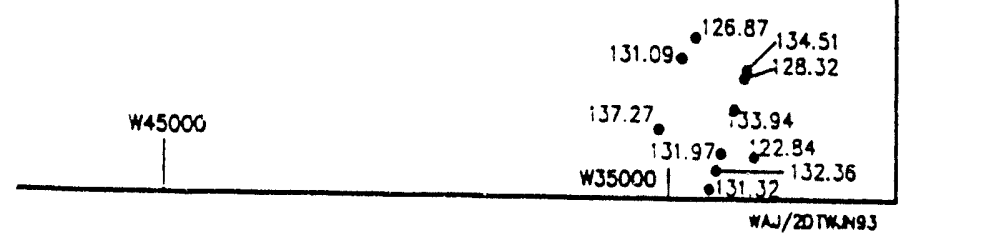

Figure 9 


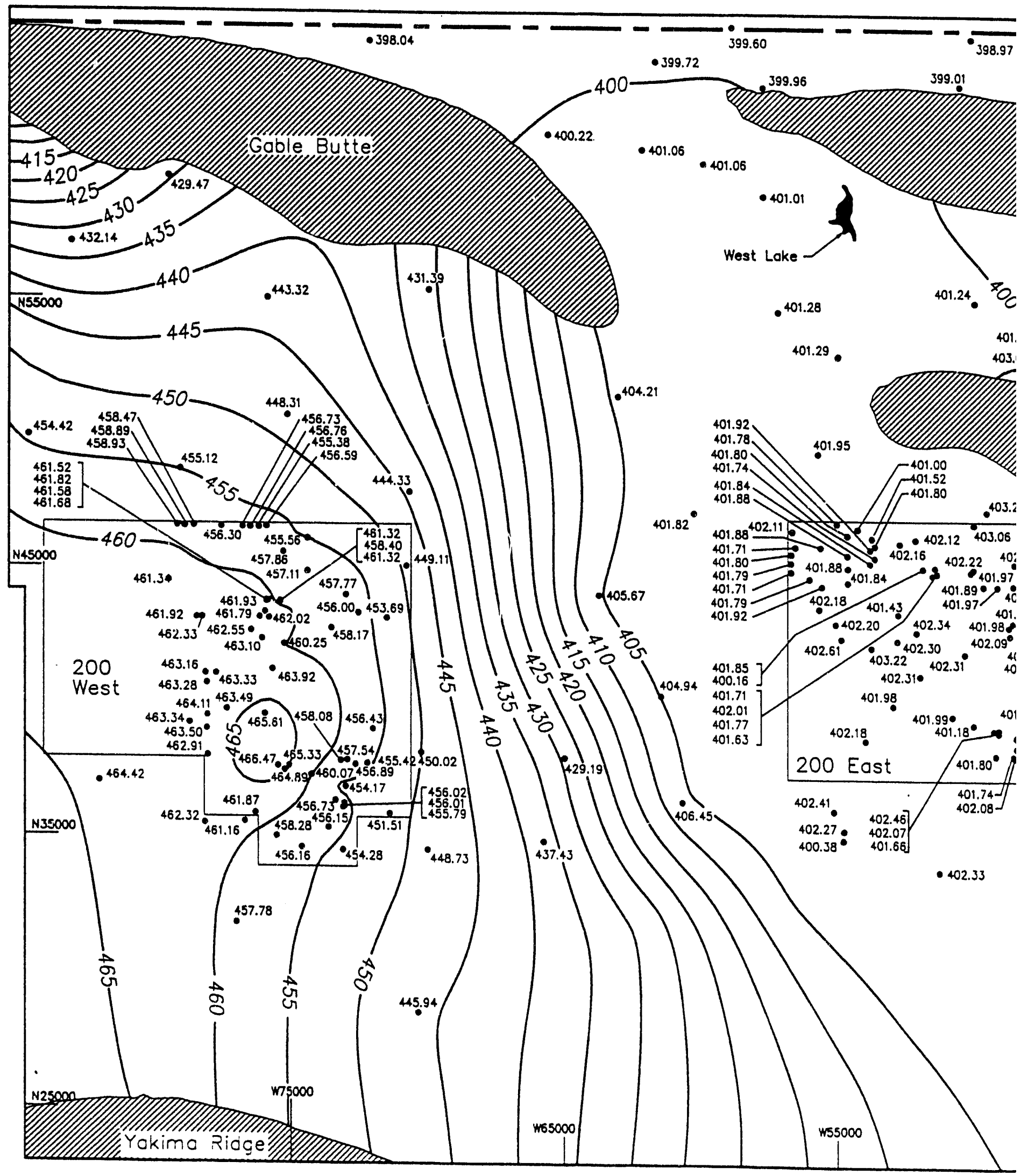




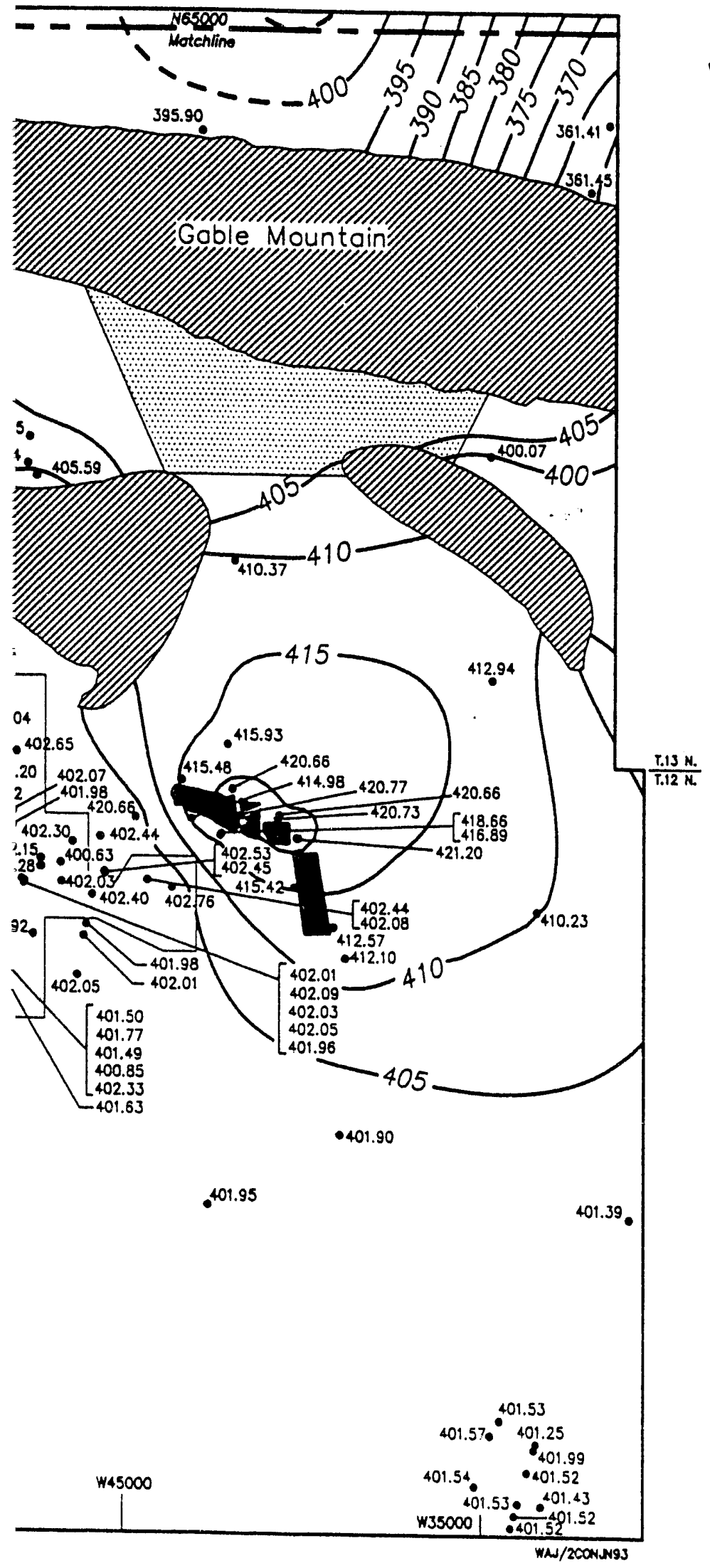

\section{Areas Water Table Elevation June 1993}

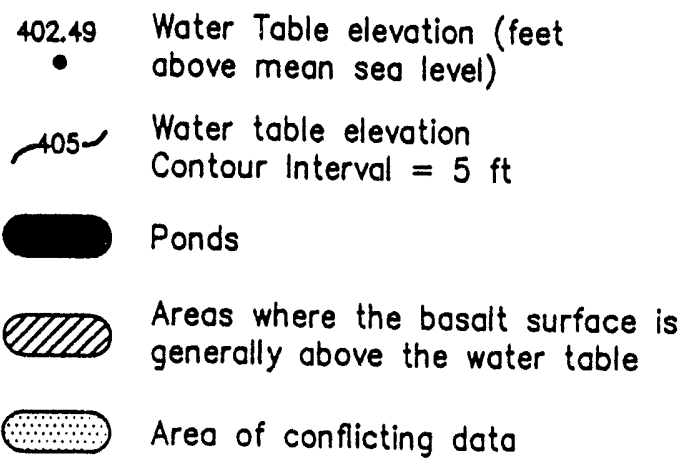

The 200 Areas water table elevation map has been prepared by the Geohydrologic Engineering Function, Westinghouse Honford Company.

Note: To convert to metric, multiply elevation ( $\mathrm{ft}$ ) by 0.3048 to obtain elevation $(m)$.

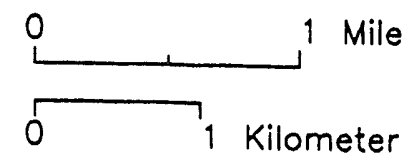

g 


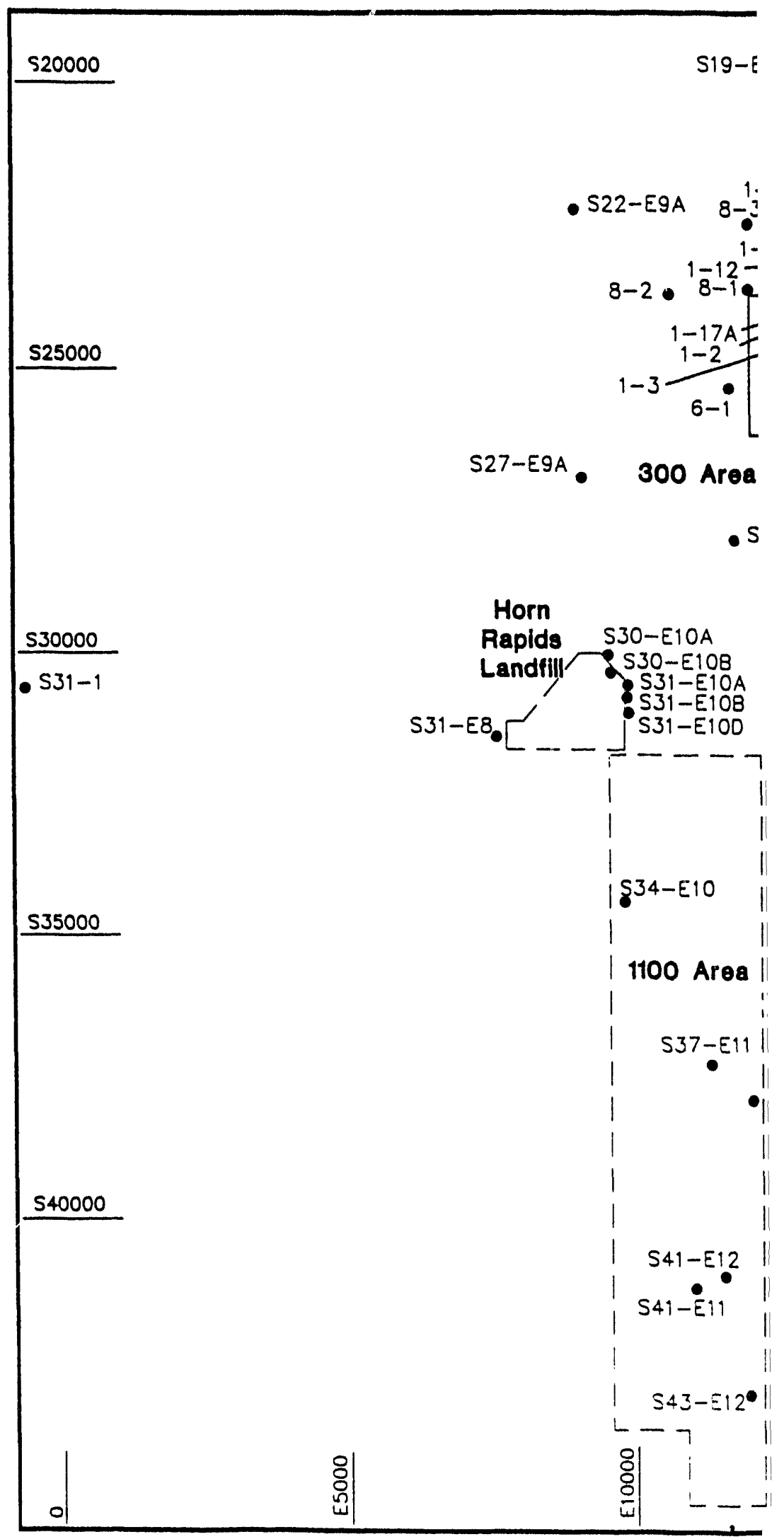


WHC-EP-0394-7

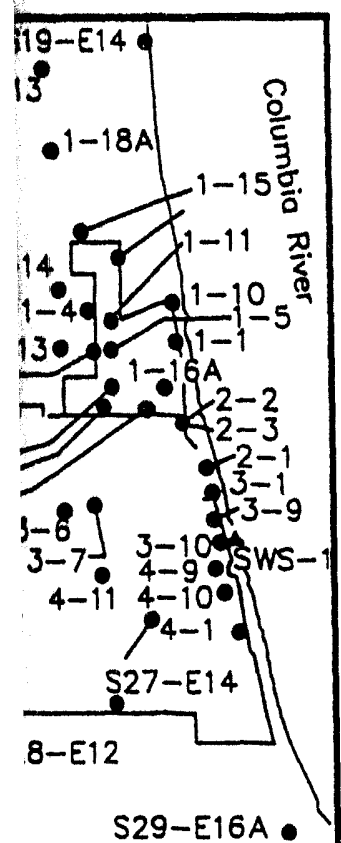

S30-E15A

- S32-E13A

S32-E13日

$30-47-188^{\circ}$

S34-E15

- $S 36-E 13 A$

S36-E12B

$537-E 14$

:38-E12A

S40-E14

- S41-E13A

\section{0/1100 Area \\ Index Map \\ June 1993}

3-1 Identification of monitoring well

- used to prepore mop. Well Numbers starting with $S$ are prefixed by 699- and well numbers storting with a single digit are prefixed by 399-.

- River stage recorder

The 300 Areo index mop has been prepared by the Geohydrologic Engineering Function, Westinghouse Hanford Company.

Note: To convert to metric, multiply elevation ( $\mathrm{ft}$ ) by 0.3048 to obtain elevation (m).

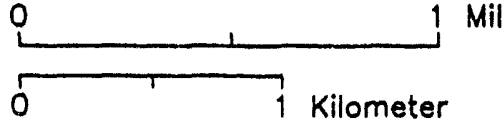




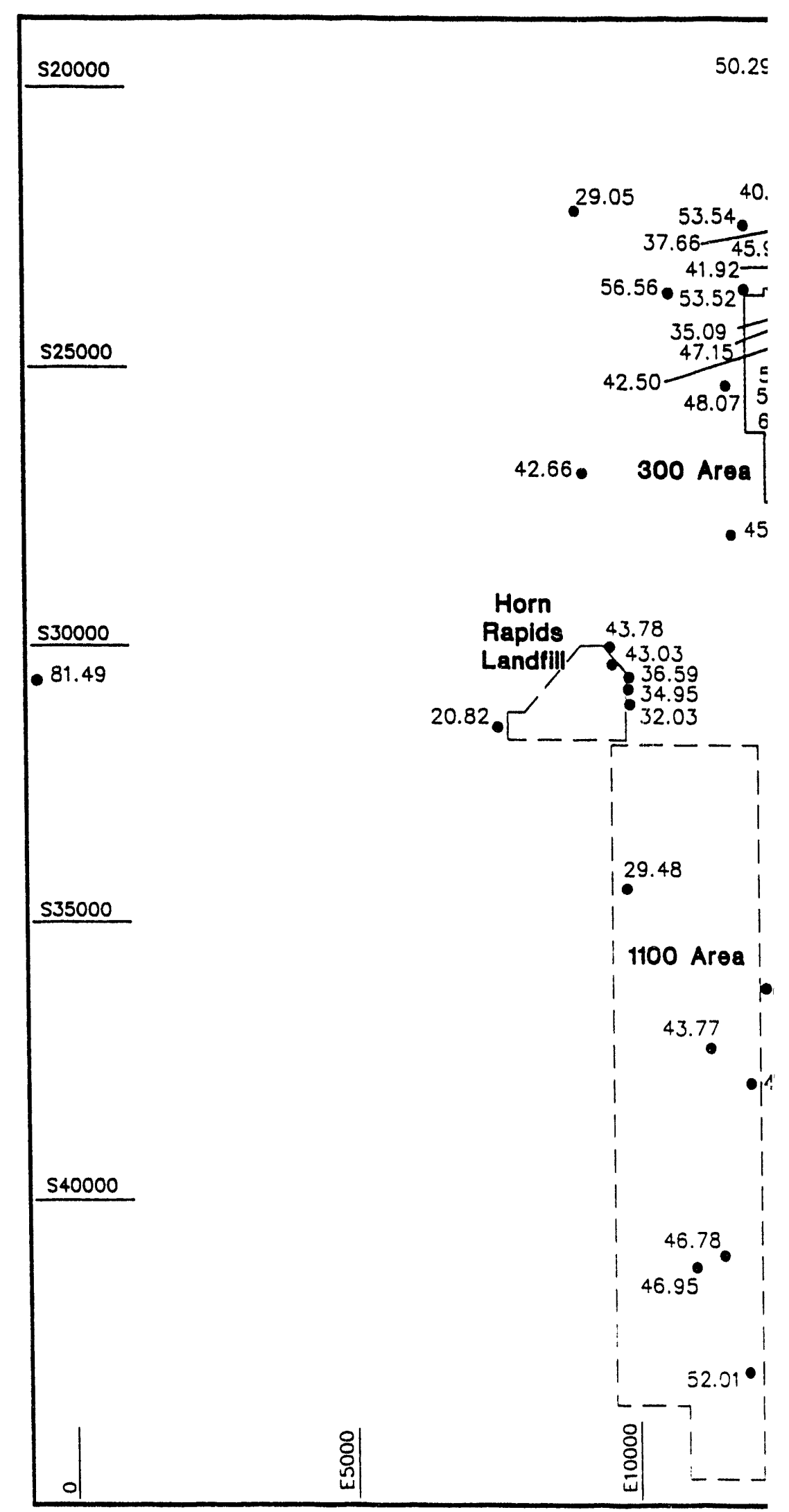


WHC-EP-0394-7

\subsection{6 \\ 300/1100 Area \\ Depth-to-Water Map \\ June 1993}

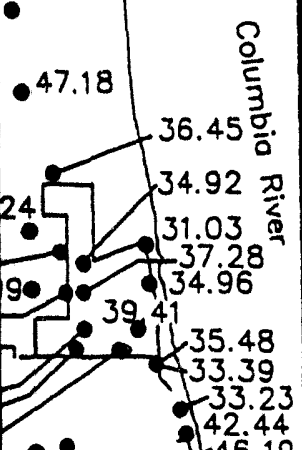

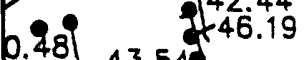

43.540

$2.49^{\circ} 40.416$

53.7937 .02 57.89

21

$38.34 \bullet$

57.77

$\bullet 43.58$

47.75 $32.88^{\circ}$

$52.31 \bullet$

$-43.66$

42.98

$$
51.46
$$

7.84

.41 .05

$\bullet 51.27$
53.19 Depth to water in feet, as

meosured from well reference

mark (generally top of casing)

to groundwater surface.

The 300 areo depth-to-water map has been

prepored by the Geohydrologic Engineering

Function, Westinghouse Hanford Company.

Note: To convert to metric, multiply elevation (ft) by 0.3048 to obtain elevation $(m)$.

0 1 Mile

0

Kilometer 


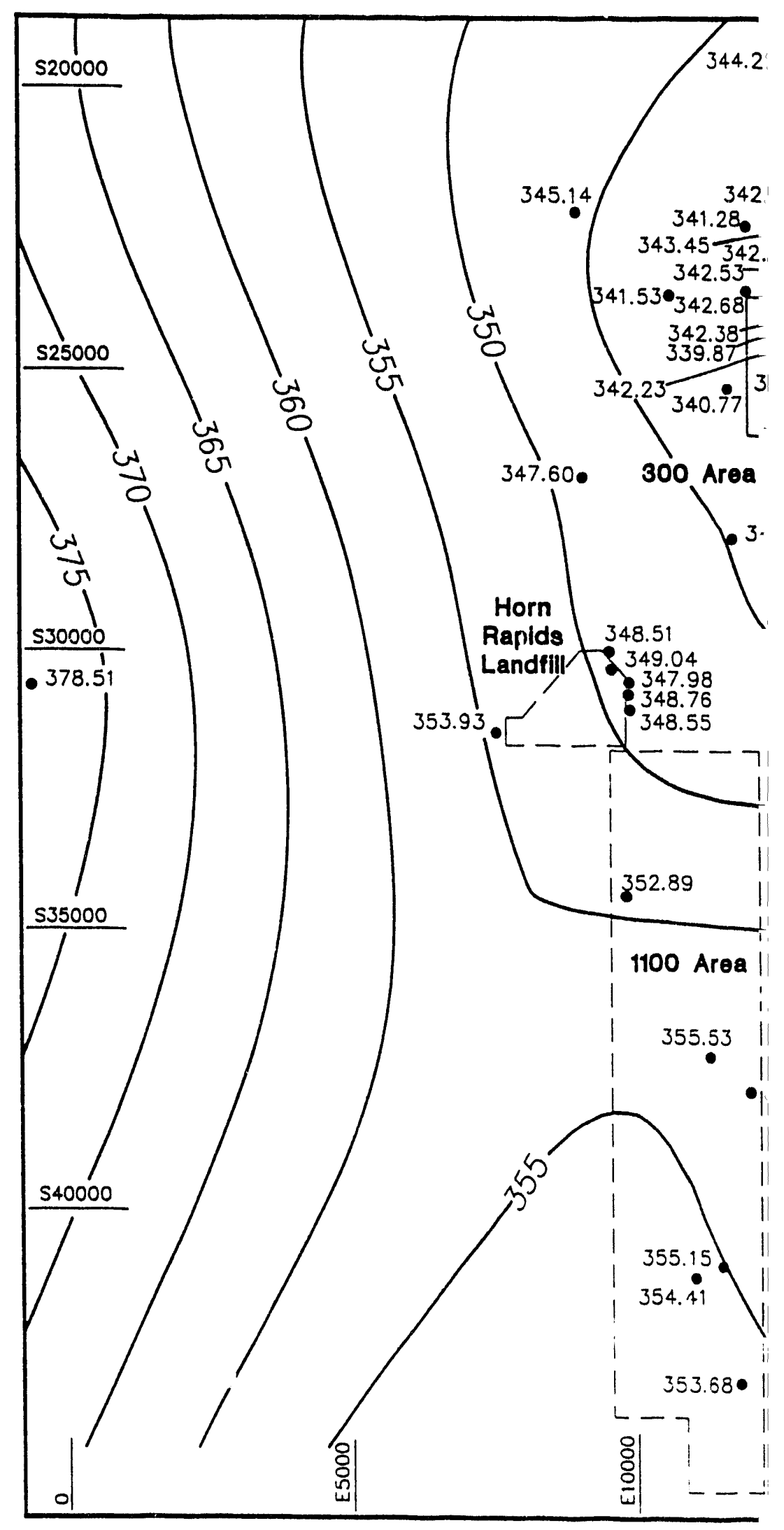


WHC-EP-0394-7
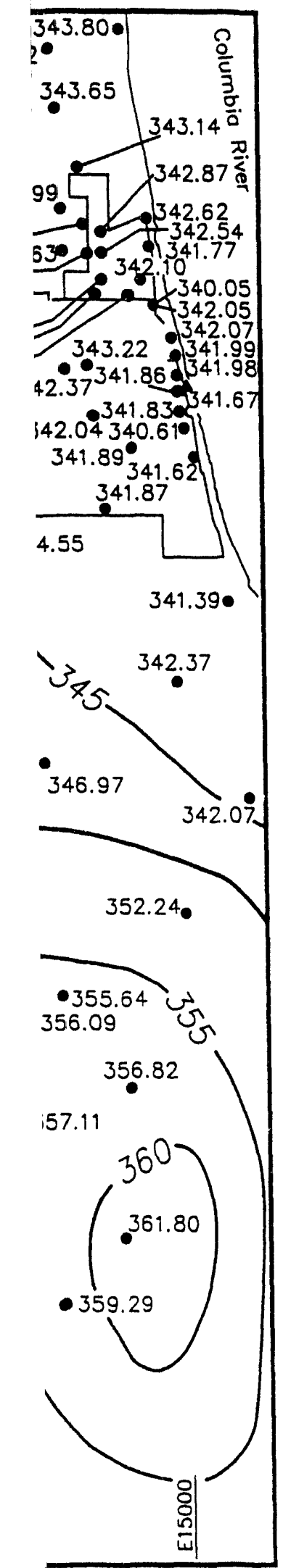

WAJ/3CONJN93

\section{0/1100 Area \\ Water Table Map June 1993}

\author{
354.19 Water table elevation (feet \\ above meon seo level) \\ - Average river elevation-day of 300 Area \\ woter level measurement \\ -350 - Water table elevation \\ Contour Interval $=5 \mathrm{ft}$ \\ The 300 Area water table mop has been \\ prepared by the Geohydrologic Engineering \\ Function, Westinghouse Hanford Company. \\ Note: To convert to metric, multiply \\ elevation ( $\mathrm{ft}$ ) by 0.3048 to obtain \\ elevation $(\mathrm{m})$.

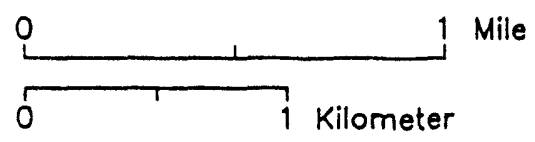

Figure 13 
WHC-EP-0394-7

APPENDIX A

JUNE 1993 WATER LEVEL MEASUREMENT DATA 
WHC-EP-0394-7

Appendix A: June 1993 Water Level Measurement Data (Sheet 1 of 22)

\begin{tabular}{|c|c|c|c|c|}
\hline \multirow{2}{*}{ Well } & \multirow{2}{*}{ Notes } & \multirow{2}{*}{$\begin{array}{l}\text { Depth to } \\
\text { Water, ft }\end{array}$} & \multicolumn{2}{|c|}{ Elevation, ft above ms l } \\
\hline & & & Adjusted Casing & Water Level \\
\hline $\begin{array}{l}199-B 3-1 \\
199-B 4-1 \\
199-B 4-3 \\
199-B 4-4 \\
199-B 4-6\end{array}$ & $N$ & $\begin{array}{l}46.27 \\
64.06 \\
64.59 \\
77.45 \\
84.44\end{array}$ & $\begin{array}{l}439.19 \\
461.19 \\
461.75 \\
473.54 \\
481.92\end{array}$ & $\begin{array}{l}392.92 \\
397.13 \\
397.16 \\
396.09 \\
397.48\end{array}$ \\
\hline $\begin{array}{l}199-B 5-1 \\
199-B 9-1 \\
199-D 2-5 \\
199-D 5-12 \\
199-D 5-13\end{array}$ & N & $\begin{array}{l}58.53 \\
98.76 \\
75.17 \\
85.12 \\
86.83\end{array}$ & $\begin{array}{l}455.58 \\
496.12 \\
460.30 \\
469.69 \\
471.49\end{array}$ & $\begin{array}{l}397.05 \\
397.36 \\
385.13 \\
384.57 \\
384.67\end{array}$ \\
\hline $\begin{array}{l}199-D 8-3 \\
199-D 8-4 \\
199-D 8-5 \\
199-D 8-6 \\
199-F 5-1\end{array}$ & $N$ & $\begin{array}{l}66.63 \\
83.37 \\
69.13 \\
91.17 \\
35.19\end{array}$ & $\begin{array}{l}449.06 \\
468.13 \\
452.48 \\
475.90 \\
406.56\end{array}$ & $\begin{array}{l}382.43 \\
384.76 \\
383.35 \\
384.73 \\
371.37\end{array}$ \\
\hline $\begin{array}{l}199-F 5-3 \\
199-F 5-4 \\
199-F 5-6 \\
199-F 7-1 \\
199-F 8-1\end{array}$ & $\begin{array}{l}N \\
N \\
N\end{array}$ & $\begin{array}{l}38.24 \\
41.99 \\
43.06 \\
15.04 \\
35.16\end{array}$ & $\begin{array}{l}408.62 \\
412.12 \\
412.95 \\
389.74 \\
405.86\end{array}$ & $\begin{array}{l}370.38 \\
370.13 \\
369.89 \\
374.70 \\
370.70\end{array}$ \\
\hline $\begin{array}{l}199-F 8-2 \\
199-H 3-1 \\
199-H 3-2 A \\
199-H 3-2 B \\
199-H 3-2 C\end{array}$ & $\begin{array}{l}N \\
N \\
N \\
N \\
P\end{array}$ & $\begin{array}{l}40.15 \\
46.00 \\
42.32 \\
42.90 \\
42.95\end{array}$ & $\begin{array}{l}410.74 \\
422.02 \\
418.37 \\
418.96 \\
418.78\end{array}$ & $\begin{array}{l}370.59 \\
376.02 \\
376.05 \\
376.06 \\
375.83\end{array}$ \\
\hline $\begin{array}{l}199-H 4-10 \\
199-H 4-11 \\
199-H 4-12 A \\
199-H 4-12 B \\
199-H 4-12 C\end{array}$ & $\begin{array}{l}N \\
N \\
N \\
N \\
P\end{array}$ & $\begin{array}{l}30.55 \\
44.16 \\
39.91 \\
39.94 \\
40.49\end{array}$ & $\begin{array}{l}404.44 \\
416.84 \\
413.50 \\
413.52 \\
413.52\end{array}$ & $\begin{array}{l}373.89 \\
372.68 \\
373.59 \\
373.58 \\
373.03\end{array}$ \\
\hline $\begin{array}{l}199-H 4-13 \\
199-H 4-14 \\
199-H 4-15 A \\
199-H 4-15 B \\
199-H 4-15 C Q\end{array}$ & $\begin{array}{l}N \\
N \\
N \\
N \\
P\end{array}$ & $\begin{array}{r}45.89 \\
44.72 \\
33.43 \\
33.12 \\
.01\end{array}$ & $\begin{array}{l}418.20 \\
420.59 \\
407.21 \\
406.92 \\
407.27\end{array}$ & $\begin{array}{l}372.31 \\
375.87 \\
373.78 \\
373.80 \\
407.26\end{array}$ \\
\hline
\end{tabular}


Appendix A: June 1993 Water Level Measurement Data (Sheet 2 of 22)

\begin{tabular}{|c|c|c|c|c|}
\hline \multirow{2}{*}{ Well } & \multirow{2}{*}{ Notes } & \multirow{2}{*}{$\begin{array}{l}\text { Depth to } \\
\text { Water, ft }\end{array}$} & \multicolumn{2}{|c|}{ Elevation, ft above msl } \\
\hline & & & Adjusted Casing & Water Level \\
\hline $\begin{array}{l}199-H 4-15 C R \\
199-H 4-15 C S \\
199-H 4-16 \\
199-H 4-17 \\
199-H 4-18\end{array}$ & $\begin{array}{l}P \\
P \\
N \\
N\end{array}$ & $\begin{array}{l}32.14 \\
32.14 \\
48.80 \\
43.46 \\
46.72\end{array}$ & $\begin{array}{l}407.37 \\
407.44 \\
424.23 \\
419.09 \\
421.82\end{array}$ & $\begin{array}{l}375.23 \\
375.30 \\
375.43 \\
375.63 \\
375.10\end{array}$ \\
\hline $\begin{array}{l}199-H 4-3 \\
199-H 4-4 \\
199-H 4-5 \\
199-H 4-6 \\
199-H 4-7\end{array}$ & $\begin{array}{l}N \\
N \\
N \\
N\end{array}$ & $\begin{array}{l}44.71 \\
40.18 \\
41.80 \\
43.56 \\
44.94\end{array}$ & $\begin{array}{l}420.29 \\
413.70 \\
416.21 \\
419.58 \\
420.59\end{array}$ & $\begin{array}{l}375.59 \\
373.53 \\
374.41 \\
376.02 \\
375.65\end{array}$ \\
\hline $\begin{array}{l}199-H 4-8 \\
199-H 4-9 \\
199-H 5-1 A \\
199-K-11 \\
199-K-13\end{array}$ & $\begin{array}{l}N \\
N \\
N \\
N\end{array}$ & $\begin{array}{l}44.47 \\
42.27 \\
43.68 \\
73.14 \\
73.37\end{array}$ & $\begin{array}{l}420.00 \\
418.08 \\
420.16 \\
466.55 \\
464.00\end{array}$ & $\begin{array}{l}375.53 \\
375.81 \\
376.48 \\
393.41 \\
390.63\end{array}$ \\
\hline $\begin{array}{l}199-K-18 \\
199-K-19 \\
199-K-20 \\
199-K-21 \\
199-K-22\end{array}$ & $\begin{array}{l}N \\
N\end{array}$ & $\begin{array}{l}21.62 \\
33.53 \\
34.55 \\
34.94 \\
37.54\end{array}$ & $\begin{array}{l}409.00 \\
422.17 \\
422.57 \\
421.73 \\
421.68\end{array}$ & $\begin{array}{l}387.38 \\
388.64 \\
388.02 \\
386.79 \\
384.14\end{array}$ \\
\hline $\begin{array}{l}199-K-23 \\
199-K-32 A \\
199-K-32 B \\
199-K-33 \\
199-K-34\end{array}$ & $\begin{array}{l}N \\
N \\
P \\
N\end{array}$ & $\begin{array}{l}74.17 \\
54.32 \\
47.49 \\
55.63 \\
76.53\end{array}$ & $\begin{array}{l}471.56 \\
444.02 \\
445.27 \\
443.64 \\
468.09\end{array}$ & $\begin{array}{l}397.39 \\
389.70 \\
397.78 \\
388.01 \\
391.56\end{array}$ \\
\hline $\begin{array}{l}199-K-35 \\
199-K-36 \\
199-K-37 \\
199-N-14 \\
199-N-16\end{array}$ & $N$ & $\begin{array}{l}98.69 \\
97.83 \\
54.24 \\
68.49 \\
69.54\end{array}$ & $\begin{array}{l}494.55 \\
494.07 \\
441.80 \\
453.15 \\
456.70\end{array}$ & $\begin{array}{l}395.86 \\
396.24 \\
387.56 \\
384.66 \\
387.16\end{array}$ \\
\hline $\begin{array}{l}199-N-17 \\
199-N-18 \\
199-N-19 \\
199-N-2 \\
199-N-20\end{array}$ & $\begin{array}{l}N \\
N \\
N \\
N \\
N\end{array}$ & $\begin{array}{l}75.52 \\
73.54 \\
69.19 \\
73.46 \\
70.48\end{array}$ & $\begin{array}{l}461.20 \\
458.50 \\
453.90 \\
459.83 \\
455.90\end{array}$ & $\begin{array}{l}385.68 \\
384.96 \\
384.71 \\
386.37 \\
385.42\end{array}$ \\
\hline
\end{tabular}


Appendix A: June 1993 Water Level Measurement Data (Sheet 3 of 22)

\begin{tabular}{|c|c|c|c|c|}
\hline \multirow{2}{*}{ Well } & \multirow{2}{*}{ Notes } & \multirow{2}{*}{$\begin{array}{l}\text { Depth to } \\
\text { Water, ft }\end{array}$} & \multicolumn{2}{|c|}{ Elevation, ft above msl } \\
\hline & & & Adjusted Casing & Water Level \\
\hline $\begin{array}{l}199-N-21 \\
199-N-23 \\
199-N-25 \\
199-N-26 \\
199-N-27\end{array}$ & $\begin{array}{l}Q \\
N \\
N \\
N\end{array}$ & $\begin{array}{l}79.66 \\
71.13 \\
42.29 \\
71.40 \\
61.76\end{array}$ & $\begin{array}{l}457.00 \\
456.30 \\
425.80 \\
455.80 \\
449.08\end{array}$ & $\begin{array}{l}377.34 \\
385.17 \\
383.51 \\
384.40 \\
387.32\end{array}$ \\
\hline $\begin{array}{l}199-N-28 \\
199-N-29 \\
199-N-3 \\
199-N-31 \\
199-N-32\end{array}$ & $\begin{array}{l}N \\
N \\
N \\
N \\
N\end{array}$ & $\begin{array}{l}76.38 \\
77.37 \\
73.19 \\
75.73 \\
75.03\end{array}$ & $\begin{array}{l}464.24 \\
465.25 \\
459.45 \\
462.63 \\
462.08\end{array}$ & $\begin{array}{l}387.86 \\
387.88 \\
386.26 \\
386.30 \\
387.05\end{array}$ \\
\hline $\begin{array}{l}199-N-33 \\
199-N-34 \\
199-N-41 \\
199-N-42 \\
199-N-49\end{array}$ & $\begin{array}{l}N \\
N \\
N \\
N \\
N\end{array}$ & $\begin{array}{l}73.02 \\
72.43 \\
72.00 \\
69.14 \\
65.56\end{array}$ & $\begin{array}{l}459.87 \\
459.63 \\
457.59 \\
455.14 \\
450.72\end{array}$ & $\begin{array}{l}386.85 \\
387.20 \\
385.59 \\
386.00 \\
385.16\end{array}$ \\
\hline $\begin{array}{l}199-N-50 \\
199-N-51 \\
199-N-52 \\
199-N-54 \\
199-N-55\end{array}$ & $\begin{array}{l}N \\
N \\
N \\
N \\
N\end{array}$ & $\begin{array}{l}78.65 \\
78.17 \\
75.52 \\
70.76 \\
71.07\end{array}$ & $\begin{array}{l}463.90 \\
462.18 \\
463.70 \\
457.51 \\
457.85\end{array}$ & $\begin{array}{l}385.25 \\
384.01 \\
388.18 \\
386.75 \\
386.78\end{array}$ \\
\hline $\begin{array}{l}199-N-56 \\
199-N-57 \\
199-N-59 \\
199-N-62 \\
199-N-63\end{array}$ & $\begin{array}{l}N \\
N \\
N \\
N\end{array}$ & $\begin{array}{l}71.68 \\
70.22 \\
72.48 \\
75.67 \\
78.94\end{array}$ & $\begin{array}{l}458.09 \\
457.76 \\
459.53 \\
463.59 \\
466.70\end{array}$ & $\begin{array}{l}386.41 \\
387.54 \\
387.05 \\
387.92 \\
387.76\end{array}$ \\
\hline $\begin{array}{l}199-N-64 \\
199-N-65 \\
199-N-67 \\
199-N-69 \\
199-N-70\end{array}$ & $\begin{array}{l}N \\
N \\
N \\
N \\
N\end{array}$ & $\begin{array}{l}67.07 \\
69.25 \\
72.32 \\
72.78 \\
68.01\end{array}$ & $\begin{array}{l}454.63 \\
456.44 \\
458.46 \\
458.84 \\
454.21\end{array}$ & $\begin{array}{l}387.56 \\
387.19 \\
386.14 \\
386.06 \\
386.20\end{array}$ \\
\hline $\begin{array}{l}199-N-71 \\
199-N-72 \\
199-N-73 \\
199-N-74 \\
199-N-80\end{array}$ & $\begin{array}{l}N \\
N \\
N \\
P\end{array}$ & $\begin{array}{l}73.40 \\
69.23 \\
74.37 \\
68.27 \\
74.52\end{array}$ & $\begin{array}{l}463.04 \\
458.99 \\
463.28 \\
457.66 \\
457.69\end{array}$ & $\begin{array}{l}389.64 \\
389.76 \\
388.91 \\
389.39 \\
383.17\end{array}$ \\
\hline
\end{tabular}


Appendix A: June 1993 Water Level Measurement Data (Sheet 4 of 22)

\begin{tabular}{|c|c|c|c|c|}
\hline \multirow{2}{*}{ Well } & \multirow{2}{*}{ Notes } & \multirow{2}{*}{$\begin{array}{l}\text { Depth to } \\
\text { Water, ft }\end{array}$} & \multicolumn{2}{|c|}{ Elevation, ft above ms 1} \\
\hline & & & Adjusted Casing & Water Level \\
\hline $\begin{array}{l}199-N-8 S \\
299-E 13-10 \\
299-E 13-12 \\
299-E 13-14 \\
299-E 16-1\end{array}$ & $Q$ & $\begin{array}{r}21.14 \\
335.43 \\
333.11 \\
342.96 \\
288.50\end{array}$ & $\begin{array}{l}404.57 \\
737.70 \\
733.49 \\
745.37 \\
695.09\end{array}$ & $\begin{array}{l}383.43 \\
402.27 \\
400.38 \\
402.41 \\
406.59\end{array}$ \\
\hline $\begin{array}{l}299-E 17-1 \\
299-E 17-10 \\
299-E 17-12 \\
299-E 17-13 \\
299-E 17-14\end{array}$ & & $\begin{array}{l}317.51 \\
312.97 \\
319.90 \\
317.42 \\
320.68\end{array}$ & $\begin{array}{l}719.17 \\
714.74 \\
721.70 \\
719.25 \\
722.18\end{array}$ & $\begin{array}{l}401.66 \\
401.77 \\
401.80 \\
401.83 \\
401.50\end{array}$ \\
\hline $\begin{array}{l}299-E 17-15 \\
299-E 17-16 \\
299-E 17-17 \\
299-E 17-18 \\
299-E 17-19\end{array}$ & N & $\begin{array}{l}320.93 \\
318.96 \\
318.18 \\
318.92 \\
318.43\end{array}$ & $\begin{array}{l}721.78 \\
720.58 \\
719.92 \\
720.65 \\
719.33\end{array}$ & $\begin{array}{l}400.85 \\
401.63 \\
401.75 \\
401.74 \\
400.90\end{array}$ \\
\hline $\begin{array}{l}299-E 17-20 \\
299-E 17-5 \\
299-E 17-6 \\
299-E 17-8 \\
299-E 17-9\end{array}$ & & $\begin{array}{l}317.16 \\
316.32 \\
318.02 \\
317.40 \\
316.16\end{array}$ & $\begin{array}{l}719.23 \\
718.65 \\
720.10 \\
718.73 \\
717.64\end{array}$ & $\begin{array}{l}402.07 \\
402.33 \\
402.08 \\
401.33 \\
401.49\end{array}$ \\
\hline $\begin{array}{l}299-E 18-1 \\
299-E 18-2 \\
299-E 18-3 \\
299-E 18-4 \\
299-E 23-1\end{array}$ & $\begin{array}{l}N \\
N\end{array}$ & $\begin{array}{l}318.19 \\
318.28 \\
319.86 \\
319.62 \\
310.79\end{array}$ & $\begin{array}{l}720.24 \\
720.24 \\
722.04 \\
721.57 \\
713.10\end{array}$ & $\begin{array}{l}402.05 \\
401.97 \\
402.18 \\
401.95 \\
402.31\end{array}$ \\
\hline $\begin{array}{l}299-E 23-2 \\
299-E 24-13 \\
299-E 24-14 \\
299-E 24-16 \\
299-E 24-18\end{array}$ & & $\begin{array}{l}319.28 \\
289.10 \\
289.30 \\
315.81 \\
318.11\end{array}$ & $\begin{array}{l}721.26 \\
691.13 \\
691.31 \\
718.27 \\
719.28\end{array}$ & $\begin{array}{l}401.98 \\
402.03 \\
402.01 \\
402.46 \\
401.18\end{array}$ \\
\hline $\begin{array}{l}299-E 24-19 \\
299-E 24-2 \\
299-E 24-20 \\
299-E 24-7 \\
299-E 24-8\end{array}$ & $\begin{array}{l}N \\
N\end{array}$ & $\begin{array}{l}291.70 \\
315.24 \\
287.00 \\
314.02 \\
286.50\end{array}$ & $\begin{array}{l}693.65 \\
717.19 \\
689.28 \\
716.01 \\
688.81\end{array}$ & $\begin{array}{l}401.96 \\
401.95 \\
402.28 \\
401.99 \\
402.31\end{array}$ \\
\hline
\end{tabular}


Appendix A: June 1993 Water Level Measurement Data (Sheet 5 of 22)

\begin{tabular}{|c|c|c|c|c|}
\hline \multirow{2}{*}{ Well } & \multirow{2}{*}{ Notes } & \multirow{2}{*}{$\begin{array}{l}\text { Depth to } \\
\text { Water, ft }\end{array}$} & \multicolumn{2}{|c|}{ Elevation, ft above ms 1} \\
\hline & & & Adjusted Casing & Water Level \\
\hline $\begin{array}{l}299-E 25-1 \\
299-E 25-10 \\
299-E 25-11 \\
299-E 25-13 \\
299-E 25-15\end{array}$ & $N$ & $\begin{array}{l}288.52 \\
253.73 \\
279.27 \\
280.34 \\
287.70\end{array}$ & $\begin{array}{r}690.57 \\
655.84 \\
681.28 \\
682.43 \\
689.73\end{array}$ & $\begin{array}{l}402.05 \\
402.11 \\
402.01 \\
402.09 \\
402.03\end{array}$ \\
\hline $\begin{array}{l}299-E 25-18 \\
299-E 25-19 \\
299-E 25-2 \\
299-E 25-20 \\
299-E 25-21\end{array}$ & $\begin{array}{l}N \\
N\end{array}$ & $\begin{array}{l}276.92 \\
275.22 \\
273.20 \\
274.35 \\
274.90\end{array}$ & $\begin{array}{l}679.05 \\
677.20 \\
675.45 \\
676.30 \\
677.27\end{array}$ & $\begin{array}{l}402.13 \\
401.98 \\
402.25 \\
401.95 \\
402.37\end{array}$ \\
\hline $\begin{array}{l}299-E 25-24 \\
299-E 25-25 \\
299-E 25-26 \\
? 99-E 25-27 \\
239-E 25-28\end{array}$ & $N$ & $\begin{array}{l}277.30 \\
266.66 \\
266.12 \\
273.64 \\
259.91\end{array}$ & $\begin{array}{l}679.55 \\
669.42 \\
668.52 \\
676.08 \\
662.44\end{array}$ & $\begin{array}{l}402.25 \\
402.76 \\
402.40 \\
402.44 \\
402.53\end{array}$ \\
\hline $\begin{array}{l}299-E 25-29 P \\
299-E 25-29 Q \\
299-E 25-30 Q \\
299-E 25-31 \\
299-E 25-32 P\end{array}$ & $\begin{array}{l}N \\
N \\
N \\
Q\end{array}$ & $\begin{array}{l}270.75 \\
270.18 \\
276.02 \\
272.20 \\
267.60\end{array}$ & $\begin{array}{l}673.06 \\
672.62 \\
677.91 \\
672.53 \\
670.04\end{array}$ & $\begin{array}{l}402.31 \\
402.44 \\
401.89 \\
400.33 \\
402.44\end{array}$ \\
\hline $\begin{array}{l}299-E 25-32 Q \\
299-E 25-34 \\
299-E 25-35 \\
299-E 25-36 \\
299-E 25-37\end{array}$ & & $\begin{array}{l}267.96 \\
260.42 \\
272.25 \\
305.47 \\
271.32\end{array}$ & $\begin{array}{l}670.04 \\
662.87 \\
674.39 \\
707.39 \\
673.29\end{array}$ & $\begin{array}{l}402.08 \\
402.45 \\
402.14 \\
401.92 \\
401.97\end{array}$ \\
\hline $\begin{array}{l}299-E 25-33 \\
299-E 25-39 \\
299-E 25-4 \\
299-E 25-40 \\
299-E 25-41\end{array}$ & $\begin{array}{l}N \\
N\end{array}$ & $\begin{array}{l}271.20 \\
268.30 \\
258.76 \\
263.57 \\
268.99\end{array}$ & $\begin{array}{l}673.52 \\
671.23 \\
659.39 \\
665.71 \\
671.26\end{array}$ & $\begin{array}{l}402.32 \\
402.93 \\
400.63 \\
402.15 \\
402.28\end{array}$ \\
\hline $\begin{array}{l}299-E 25-42 \\
299-E 25-43 \\
299-E 25-46 \\
299-E 25-6 \\
299-E 25-9\end{array}$ & $\begin{array}{l}N \\
N \\
N \\
Q \\
Q\end{array}$ & $\begin{array}{l}281.07 \\
247.18 \\
292.49 \\
258.80 \\
254.13\end{array}$ & $\begin{array}{l}683.29 \\
649.89 \\
694.81 \\
658.31 \\
654.17\end{array}$ & $\begin{array}{l}402.22 \\
402.71 \\
402.33 \\
399.51 \\
400.04\end{array}$ \\
\hline
\end{tabular}


Appendix A: June 1993 Water Level Measurement Data (Sheet 6 of 22)

\begin{tabular}{|c|c|c|c|c|}
\hline \multirow{2}{*}{ Well } & \multirow{2}{*}{ Notes } & \multirow{2}{*}{$\begin{array}{l}\text { Depth to } \\
\text { Water, ft }\end{array}$} & \multicolumn{2}{|c|}{ Elevation, ft above ms 1} \\
\hline & & & Adjusted Casing & Water Level \\
\hline $\begin{array}{l}299-E 26-1 \\
299-E 26-10 \\
299-E 26-11 \\
299-E 26-12 \\
299-E 26-13\end{array}$ & $\begin{array}{l}N \\
N \\
N \\
N\end{array}$ & $\begin{array}{l}214.60 \\
199.20 \\
194.58 \\
227.85 \\
202.19\end{array}$ & $\begin{array}{l}617.25 \\
601.73 \\
599.94 \\
630.74 \\
605.02\end{array}$ & $\begin{array}{l}402.65 \\
402.53 \\
405.36 \\
402.89 \\
402.83\end{array}$ \\
\hline $\begin{array}{l}299-E 26-2 \\
299-E 26-4 \\
299-E 26-8 \\
299-E 26-9 \\
299-E 27-1\end{array}$ & $\begin{array}{l}N \\
N \\
N\end{array}$ & $\begin{array}{l}232.86 \\
245.46 \\
234.45 \\
200.63 \\
280.38\end{array}$ & $\begin{array}{l}635.30 \\
647.76 \\
619.85 \\
603.15 \\
682.55\end{array}$ & $\begin{array}{l}402.44 \\
402.30 \\
385.40 \\
402.52 \\
402.17\end{array}$ \\
\hline $\begin{array}{l}299-E 27-10 \\
299-E 27-11 \\
299-E 27-12 \\
299-E 27-13 \\
299-E 27-14\end{array}$ & & $\begin{array}{l}222.27 \\
241.58 \\
258.98 \\
266.91 \\
256.36\end{array}$ & $\begin{array}{l}624.47 \\
643.29 \\
660.95 \\
668.99 \\
658.34\end{array}$ & $\begin{array}{l}402.20 \\
401.71 \\
401.98 \\
402.09 \\
401.98\end{array}$ \\
\hline $\begin{array}{l}299-E 27-15 \\
299-E 27-16 \\
299-E 27-17 \\
299-E 27-18 \\
299-E 27-19\end{array}$ & $\begin{array}{l}N \\
N \\
N\end{array}$ & $\begin{array}{l}251.06 \\
250.37 \\
232.62 \\
248.10 \\
249.89\end{array}$ & $\begin{array}{l}652.67 \\
652.13 \\
634.72\end{array}$ & $\begin{array}{l}401.62 \\
401.77 \\
402.10\end{array}$ \\
\hline $\begin{array}{l}299-E 27-7 \\
299-E 27-8 \\
299-E 27-9 \\
299-E 28-12 \\
299-E 28-17\end{array}$ & & $\begin{array}{l}232.60 \\
235.94 \\
227.24 \\
305.99 \\
305.34\end{array}$ & $\begin{array}{l}634.67 \\
637.83 \\
629.21 \\
708.60 \\
708.56\end{array}$ & $\begin{array}{l}402.07 \\
401.89 \\
401.97 \\
402.61 \\
403.22\end{array}$ \\
\hline $\begin{array}{l}299-E 28-18 \\
299-E 28-26 \\
299-E 28-27 \\
299-E 28-28 \\
299-E 28-4\end{array}$ & & $\begin{array}{l}290.40 \\
285.34 \\
278.53 \\
284.76 \\
289.21\end{array}$ & $\begin{array}{l}692.58 \\
687.26 \\
680.37 \\
686.55 \\
691.55\end{array}$ & $\begin{array}{l}402.18 \\
401.92 \\
401.84 \\
401.79 \\
402.34\end{array}$ \\
\hline $\begin{array}{l}299-E 28-6 \\
299-E 28-7 \\
299-E 28-9 \\
299-E 32-1 \\
299-E 32-2\end{array}$ & & $\begin{array}{l}297.81 \\
284.48 \\
298.57 \\
254.06 \\
268.35\end{array}$ & $\begin{array}{l}700.11 \\
685.91 \\
700.77 \\
656.17 \\
670.06\end{array}$ & $\begin{array}{l}402.30 \\
401.43 \\
402.20 \\
402.11 \\
401.71\end{array}$ \\
\hline
\end{tabular}


Appendix. A: June 1993 Water Level Measurement Data (Sheet 7 of 22)

\begin{tabular}{|c|c|c|c|c|}
\hline \multirow{2}{*}{ Well } & \multirow{2}{*}{ Notes } & \multirow{2}{*}{$\begin{array}{l}\text { Depth to } \\
\text { Water, ft }\end{array}$} & \multicolumn{2}{|c|}{ Elevation, ft above ms 1} \\
\hline & & & Adjusted Casing & Water Level \\
\hline $\begin{array}{l}299-E 32-3 \\
299-E 32-4 \\
299-E 32-5 \\
299-E 32-6 \\
299-E 32-7\end{array}$ & $\begin{array}{l}N \\
N\end{array}$ & $\begin{array}{l}274.71 \\
284.17 \\
280.35 \\
265.54 \\
256.44\end{array}$ & $\begin{array}{l}676.51 \\
685.88 \\
682.14 \\
667.45 \\
658.42\end{array}$ & $\begin{array}{l}401.80 \\
401.71 \\
401.79 \\
401.91 \\
401.98\end{array}$ \\
\hline $\begin{array}{l}299-E 32-8 \\
299-E 32-9 \\
299-E 33-1 \\
299-E 33-12 \\
299-E 33-13\end{array}$ & $\begin{array}{l}N \\
N \\
N \\
P \\
N\end{array}$ & $\begin{array}{l}243.69 \\
241.35 \\
233.01 \\
220.86 \\
229.10\end{array}$ & $\begin{array}{l}645.59 \\
643.33 \\
632.11 \\
623.45 \\
628.39\end{array}$ & $\begin{array}{l}401.90 \\
401.98 \\
399.10 \\
402.59 \\
399.29\end{array}$ \\
\hline $\begin{array}{l}299-E 33-14 \\
299-E 33-15 \\
299-E 33-17 \\
299-E 33-18 \\
299-E 33-2\end{array}$ & $\begin{array}{l}N \\
N \\
N\end{array}$ & $\begin{array}{l}219.93 \\
225.13 \\
229.57 \\
249.63 \\
229.85\end{array}$ & $\begin{array}{l}622.05 \\
627.29 \\
631.65 \\
651.86 \\
630.00\end{array}$ & $\begin{array}{l}402.12 \\
402.16 \\
402.08 \\
402.23 \\
400.15\end{array}$ \\
\hline $\begin{array}{l}299-E 33-21 \\
299-E 33-24 \\
299-E 33-26 \\
299-E 33-28 \\
299-E 33-29\end{array}$ & & $\begin{array}{l}266.55 \\
236.45 \\
231.77 \\
262.35 \\
271.89\end{array}$ & $\begin{array}{l}668.39 \\
637.97 \\
632.77 \\
664.23 \\
673.77\end{array}$ & $\begin{array}{l}401.84 \\
401.52 \\
401.00 \\
401.88 \\
401.88\end{array}$ \\
\hline $\begin{array}{l}299-E 33-3 \\
299-E 33-30 \\
299-E 33-31 \\
299-E 33-32 \\
299-E 33-33\end{array}$ & N & $\begin{array}{l}233.34 \\
261.82 \\
245.49 \\
258.09 \\
238.32\end{array}$ & $\begin{array}{l}630.62 \\
663.70 \\
647.28 \\
659.83 \\
640.17\end{array}$ & $\begin{array}{l}397.28 \\
401.88 \\
401.80 \\
401.74 \\
401.85\end{array}$ \\
\hline $\begin{array}{l}299-E 33-34 \\
299-E 33-35 \\
299-E 33-36 \\
299-E 33-37 \\
299-E 33-4\end{array}$ & $N$ & $\begin{array}{l}231.55 \\
241.21 \\
246.51 \\
251.00 \\
229.68\end{array}$ & $\begin{array}{l}633.33 \\
643.01 \\
646.67 \\
553.01 \\
629.84\end{array}$ & $\begin{array}{l}401.78 \\
401.80 \\
400.16 \\
402.01 \\
400.16\end{array}$ \\
\hline $\begin{array}{l}299-E 33-41 \\
299-E 33-42 \\
299-E 33-43 \\
299-E 33-5 \\
299-E 33-7\end{array}$ & $\begin{array}{l}N \\
N \\
N \\
N \\
N\end{array}$ & $\begin{array}{l}252.93 \\
252.26 \\
261.31 \\
235.42 \\
226.97\end{array}$ & $\begin{array}{l}654.95 \\
654.30 \\
662.68 \\
634.72 \\
627.82\end{array}$ & $\begin{array}{l}402.02 \\
402.05 \\
401.38 \\
399.30 \\
400.85\end{array}$ \\
\hline
\end{tabular}


Appendix A: June 1993 Water Level Measurement Data (Sheet 8 of 22)

\begin{tabular}{|c|c|c|c|c|}
\hline \multirow{2}{*}{ Well } & \multirow{2}{*}{ Notes } & \multirow{2}{*}{$\begin{array}{l}\text { Depth to } \\
\text { Water, ft }\end{array}$} & \multicolumn{2}{|c|}{ Elevation, $\mathrm{ft}$ above msl } \\
\hline & & & Adjusted Casing & Water Level \\
\hline $\begin{array}{l}299-E 33-8 \\
299-E 34-1 \\
299-E 34-10 \\
299-E 34-2 \\
299-E 34-3\end{array}$ & $N$ & $\begin{array}{l}248.81 \\
227.23 \\
237.67 \\
228.83 \\
209.48\end{array}$ & $\begin{array}{l}650.73 \\
629.45 \\
639.78 \\
630.80 \\
611.52\end{array}$ & $\begin{array}{l}401.92 \\
402.22 \\
402.11 \\
401.97 \\
402.04\end{array}$ \\
\hline $\begin{array}{l}299-E 34-5 \\
299-E 34-8 \\
299-E 34-9 \\
299-E 35-2 \\
299-W 10-10\end{array}$ & $\begin{array}{l}N \\
N\end{array}$ & $\begin{array}{l}187.73 \\
238.89 \\
226.67 \\
199.84 \\
213.83\end{array}$ & $\begin{array}{l}590.79 \\
640.52 \\
628.69 \\
602.36 \\
675.35\end{array}$ & $\begin{array}{l}403.06 \\
401.63 \\
402.02 \\
402.52 \\
461.52\end{array}$ \\
\hline $\begin{array}{l}299-W 10-11 \\
299-W 10-12 \\
299-W 10-13 \\
299-W 10-14 \\
299-W 10-15\end{array}$ & & $\begin{array}{l}213.44 \\
213.43 \\
236.71 \\
237.51 \\
213.97\end{array}$ & $\begin{array}{l}675.26 \\
675.01 \\
699.04 \\
699.43 \\
675.64\end{array}$ & $\begin{array}{l}461.82 \\
461.58 \\
462.33 \\
461.92 \\
461.68\end{array}$ \\
\hline $\begin{array}{l}299-W 10-16 \\
299-W 10-17 \\
299-W 10-18 \\
299-W 10-2 \\
299-W 10-3\end{array}$ & $\begin{array}{l}N \\
N\end{array}$ & $\begin{array}{l}210.74 \\
208.68 \\
208.19 \\
212.46 \\
209.79\end{array}$ & $\begin{array}{l}672.76 \\
670.84 \\
670.93 \\
674.25 \\
671.72\end{array}$ & $\begin{array}{l}462.02 \\
462.17 \\
462.74 \\
461.79 \\
461.93\end{array}$ \\
\hline $\begin{array}{l}299-W 10-5 \\
299-W 10-8 \\
299-W 10-9 \\
299-W 11-10 \\
299-W 11-12\end{array}$ & $\begin{array}{l}N \\
N\end{array}$ & $\begin{array}{l}210.76 \\
218.99 \\
213.27 \\
275.20 \\
217.91\end{array}$ & $\begin{array}{l}673.31 \\
680.31 \\
674.93 \\
728.89 \\
679.52\end{array}$ & $\begin{array}{l}462.55 \\
461.32 \\
461.66 \\
453.69 \\
461.61\end{array}$ \\
\hline $\begin{array}{l}299-W 11-14 \\
299-W 11-19 \\
299-W 11-23 \\
299-W 11-24 \\
299-W 11-27\end{array}$ & $N$ & $\begin{array}{l}257.39 \\
249.89 \\
227.28 \\
226.11 \\
223.70\end{array}$ & $\begin{array}{l}715.16 \\
707.00 \\
685.68 \\
687.43 \\
685.27\end{array}$ & $\begin{array}{l}457.77 \\
457.11 \\
458.40 \\
461.32 \\
461.57\end{array}$ \\
\hline $\begin{array}{l}299-W 11-31 \\
299-W 11-6 \\
299-W 11-9 \\
299-W 12-1 \\
299-W 14-1\end{array}$ & $N$ & $\begin{array}{l}249.18 \\
258.06 \\
266.94 \\
277.35 \\
211.61\end{array}$ & $\begin{array}{l}716.23 \\
722.94 \\
726.46 \\
671.86\end{array}$ & $\begin{array}{l}458.17 \\
456.00 \\
449.11 \\
460.25\end{array}$ \\
\hline
\end{tabular}


Appendix A: June 1993 Water Level Measurement Data (Sheet 9 of 22)

\begin{tabular}{|c|c|c|c|c|}
\hline \multirow{2}{*}{ Well } & \multirow{2}{*}{ Notes } & \multirow{2}{*}{$\begin{array}{l}\text { Depth to } \\
\text { Water, ft }\end{array}$} & \multicolumn{2}{|c|}{ Elevation, ft above msl } \\
\hline & & & Adjusted Casing & Water Level \\
\hline $\begin{array}{l}299-W 14-12 \\
299-W 15-10 \\
299-W 15-12 \\
299-W 15-13 \\
299-W 15-15\end{array}$ & $\begin{array}{l}N \\
N \\
N \\
N\end{array}$ & $\begin{array}{l}206.85 \\
213.18 \\
206.97 \\
207.04 \\
234.54\end{array}$ & $\begin{array}{l}670.52 \\
676.00 \\
670.07 \\
670.12 \\
697.96\end{array}$ & $\begin{array}{l}463.67 \\
462.82 \\
463.10 \\
463.08 \\
463.42\end{array}$ \\
\hline $\begin{array}{l}299-W 15-16 \\
299-W 15-17 \\
299-W 15-18 \\
299-W 15-19 \\
299-W 15-20\end{array}$ & $\begin{array}{l}N \\
N\end{array}$ & $\begin{array}{l}220.88 \\
220.93 \\
221.60 \\
228.27 \\
235.20\end{array}$ & $\begin{array}{l}684.89 \\
684.64 \\
685.71 \\
691.60 \\
698.36\end{array}$ & $\begin{array}{l}464.01 \\
463.71 \\
464.11 \\
463.33 \\
463.16\end{array}$ \\
\hline $\begin{array}{l}299-W 15-22 \\
299-W 15-23 \\
299-W 15-24 \\
299-W 15-3 \\
299-W 15-4\end{array}$ & $\begin{array}{l}N \\
N \\
N\end{array}$ & $\begin{array}{l}205.99 \\
236.21 \\
235.88 \\
207.51 \\
198.08\end{array}$ & $\begin{array}{l}670.77 \\
699.49 \\
699.37 \\
670.50 \\
662.00\end{array}$ & $\begin{array}{l}464.78 \\
463.28 \\
463.49 \\
462.99 \\
463.92\end{array}$ \\
\hline $\begin{array}{l}299-W 15-5 \\
299-W 18-15 \\
299-W 18-21 \\
299-W 18-22 \\
299-W 18-23\end{array}$ & $\begin{array}{l}N \\
N\end{array}$ & $\begin{array}{l}205.07 \\
196.54 \\
205.15 \\
205.58 \\
233.31\end{array}$ & $\begin{array}{l}670.68 \\
660.96 \\
668.62 \\
668.49 \\
696.81\end{array}$ & $\begin{array}{l}465.61 \\
464.42 \\
463.47 \\
462.91 \\
463.50\end{array}$ \\
\hline $\begin{array}{l}299-W 18-24 \\
299-W 18-25 \\
299-W 18-26 \\
299-W 18-27 \\
299-W 18-28\end{array}$ & $\begin{array}{l}N \\
N \\
N \\
N\end{array}$ & $\begin{array}{l}220.10 \\
200.83 \\
235.56 \\
226.70 \\
216.23\end{array}$ & $\begin{array}{l}684.35 \\
666.04 \\
699.05 \\
690.25 \\
679.99\end{array}$ & $\begin{array}{l}464.25 \\
465.22 \\
463.49 \\
463.55 \\
463.76\end{array}$ \\
\hline $\begin{array}{l}299-W 18-29 \\
299-W 18-30 \\
299-W 18-31 \\
299-W 19-1 \\
299-W 19-12\end{array}$ & $\begin{array}{l}Q \\
N \\
N \\
P\end{array}$ & $\begin{array}{l}126.78 \\
208.27 \\
199.01 \\
207.30 \\
208.12\end{array}$ & $\begin{array}{l}674.14 \\
672.84 \\
664.16 \\
673.77 \\
673.25\end{array}$ & $\begin{array}{l}547.36 \\
464.57 \\
465.15 \\
466.47 \\
465.13\end{array}$ \\
\hline $\begin{array}{l}299-W 19-14 \\
299-W 19-15 \\
299-W 19-2 \\
299-W 19-20 \\
299-W 19-21\end{array}$ & $N$ & $\begin{array}{l}233.14 \\
232.48 \\
239.87 \\
235.62 \\
213.64\end{array}$ & $\begin{array}{l}693.21 \\
693.28 \\
694.04 \\
691.04 \\
678.53\end{array}$ & $\begin{array}{l}460.07 \\
460.80 \\
454.17 \\
455.42 \\
464.89\end{array}$ \\
\hline
\end{tabular}


Appendix A: June 1993 Water Level Measurement Data

(Sheet 10 of 22)

\begin{tabular}{|c|c|c|c|c|}
\hline \multirow{2}{*}{ Well } & \multirow{2}{*}{ Notes } & \multirow{2}{*}{$\begin{array}{l}\text { Depth to } \\
\text { Water, ft }\end{array}$} & \multicolumn{2}{|c|}{ Elevation, $\mathrm{ft}$ above ms 1} \\
\hline & & & Adjusted Casing & Water Level \\
\hline $\begin{array}{l}299-W 19-24 \\
299-W 19-27 \\
299-W 19-28 \\
299-W 19-29 \\
299-W 19-31\end{array}$ & $N$ & $\begin{array}{l}240.76 \\
218.32 \\
245.01 \\
244.33 \\
208.91\end{array}$ & $\begin{array}{l}696.95 \\
683.65 \\
703.09 \\
701.87 \\
674.19\end{array}$ & $\begin{array}{l}456.19 \\
465.33 \\
458.08 \\
457.54 \\
465.29\end{array}$ \\
\hline $\begin{array}{l}299-W 19-32 \\
299-W 19-4 \\
299-W 19-6 \\
299-W 19-91 \\
299-W 19-92\end{array}$ & $\begin{array}{l}N \\
N \\
P \\
P\end{array}$ & $\begin{array}{r}209.57 \\
259.09 \\
226.09 \\
90.58 \\
90.21\end{array}$ & $\begin{array}{l}674.90 \\
715.52 \\
686.60 \\
677.90 \\
677.90\end{array}$ & $\begin{array}{l}465.33 \\
456.43 \\
460.51 \\
587.32 \\
587.69\end{array}$ \\
\hline $\begin{array}{l}299-W 19-93 \\
299-W 21-1 \\
299-W 22-19 \\
299-W 22-21 \\
299-W 22-26\end{array}$ & $N$ & $\begin{array}{r}53.48 \\
247.75 \\
226.98 \\
213.84 \\
221.49\end{array}$ & $\begin{array}{l}677.43 \\
699.26 \\
681.26 \\
670.00 \\
680.30\end{array}$ & $\begin{array}{l}623.95 \\
451.51 \\
454.28 \\
456.16 \\
458.81\end{array}$ \\
\hline $\begin{array}{l}299-W 22-39 \\
299-W 22-40 \\
299-W 22-41 \\
299-W 22-42 \\
299-W 22-43\end{array}$ & $N$ & $\begin{array}{l}209.73 \\
236.47 \\
235.99 \\
235.63 \\
234.88\end{array}$ & $\begin{array}{l}668.26 \\
692.49 \\
692.00 \\
691.42 \\
691.61\end{array}$ & $\begin{array}{l}458.53 \\
456.02 \\
456.01 \\
455.79 \\
456.73\end{array}$ \\
\hline $\begin{array}{l}299-W 22-44 \\
299-W 22-45 \\
299-W 22-46 \\
299-W 22-7 \\
299-W 23-1\end{array}$ & $\begin{array}{l}N \\
N \\
N \\
N\end{array}$ & $\begin{array}{l}217.23 \\
206.91 \\
212.85 \\
231.26 \\
204.62\end{array}$ & $\begin{array}{l}678.13 \\
666.21 \\
671.18 \\
687.41 \\
666.14\end{array}$ & $\begin{array}{l}460.91 \\
459.30 \\
458.33 \\
456.15 \\
461.52\end{array}$ \\
\hline $\begin{array}{l}299-W 23-11 \\
299-W 23-12 \\
299-W 23-13 \\
299-W 23-14 \\
299-W 23-15\end{array}$ & $\begin{array}{l}N \\
N \\
N \\
N\end{array}$ & $\begin{array}{l}202.98 \\
205.30 \\
206.49 \\
203.46 \\
196.09\end{array}$ & $\begin{array}{l}664.14 \\
666.15 \\
666.33 \\
664.00 \\
655.44\end{array}$ & $\begin{array}{l}461.16 \\
460.85 \\
459.84 \\
460.54 \\
459.35\end{array}$ \\
\hline $\begin{array}{l}299-W 23-2 \\
299-W 23-3 \\
299-W 23-4 \\
299-W 23-6 \\
299-W 23-7\end{array}$ & $\begin{array}{l}N \\
N\end{array}$ & $\begin{array}{l}203.71 \\
204.62 \\
200.76 \\
208.70 \\
203.32\end{array}$ & $\begin{array}{l}663.48 \\
663.56 \\
662.63 \\
666.98 \\
663.67\end{array}$ & $\begin{array}{l}459.77 \\
458.94 \\
461.87 \\
458.28 \\
460.35\end{array}$ \\
\hline
\end{tabular}


Appendix A: June 1993 Water Level Measurement Data

(Sheet 11 of 22)

\begin{tabular}{|c|c|c|c|c|}
\hline \multirow{2}{*}{ Well } & \multirow{2}{*}{ Notes } & \multirow{2}{*}{$\begin{array}{l}\text { Depth to } \\
\text { Water, ft }\end{array}$} & \multicolumn{2}{|c|}{ Elevation, ft above msl } \\
\hline & & & Adjusted Casing & Water Level \\
\hline $\begin{array}{l}299-W 23-8 \\
299-W 26-10 \\
299-W 26-12 \\
299-W 26-7 \\
299-W 26-8\end{array}$ & $\begin{array}{l}N \\
N \\
N \\
N \\
N\end{array}$ & $\begin{array}{l}203.31 \\
213.84 \\
218.26 \\
191.88 \\
209.03\end{array}$ & $\begin{array}{l}663.95 \\
670.89 \\
675.69 \\
651.99 \\
666.57\end{array}$ & $\begin{array}{l}460.64 \\
457.06 \\
457.43 \\
460.11 \\
457.55\end{array}$ \\
\hline $\begin{array}{l}299-W 26-9 \\
299-W 6-1 \\
299-W 6-10 \\
299-W 6-11 \\
299-W 6-12\end{array}$ & $\begin{array}{l}N \\
N \\
N \\
N\end{array}$ & $\begin{array}{l}196.98 \\
246.97 \\
257.78 \\
249.96 \\
235.35\end{array}$ & $\begin{array}{l}654.42 \\
702.53\end{array}$ & $\begin{array}{l}457.44 \\
455.56\end{array}$ \\
\hline $\begin{array}{l}299-W 6-2 \\
299-W 6-3 \\
299-W 6-4 \\
299-W 6-6 \\
299-W 6-7\end{array}$ & $\begin{array}{l}N \\
N \\
N \\
N\end{array}$ & $\begin{array}{l}234.59 \\
242.95 \\
245.33 \\
271.75 \\
271.80\end{array}$ & $\begin{array}{l}692.45 \\
699.83 \\
701.25 \\
709.99 \\
710.28\end{array}$ & $\begin{array}{l}457.86 \\
456.88 \\
455.92 \\
438.24 \\
438.48\end{array}$ \\
\hline $\begin{array}{l}299-W 6-8 \\
299-W 6-9 \\
299-W 7-1 \\
299-W 7-10 \\
299-W 7-11\end{array}$ & $\begin{array}{l}N \\
N \\
N \\
N\end{array}$ & $\begin{array}{l}223.98 \\
239.32 \\
232.24 \\
229.45 \\
223.29\end{array}$ & $\begin{array}{l}694.73 \\
690.71 \\
686.66 \\
681.45\end{array}$ & $\begin{array}{l}470.75 \\
458.47 \\
457.21 \\
458.16\end{array}$ \\
\hline $\begin{array}{l}299-W 7-12 \\
299-W 7-2 \\
299-W 7-3 \\
299-W 7-5 \\
299-W 7-6\end{array}$ & $\begin{array}{l}N \\
N\end{array}$ & $\begin{array}{l}229.45 \\
218.39 \\
219.84 \\
216.32 \\
222.05\end{array}$ & $\begin{array}{l}687.93 \\
675.59 \\
676.14 \\
673.05 \\
678.64\end{array}$ & $\begin{array}{l}458.48 \\
457.20 \\
456.30 \\
456.73 \\
456.59\end{array}$ \\
\hline $\begin{array}{l}299-W 7-7 \\
299-W 7-8 \\
299-W 7-9 \\
299-W 8-1 \\
299-W 9-1\end{array}$ & & $\begin{array}{l}218.18 \\
231.97 \\
233.20 \\
242.40 \\
276.39\end{array}$ & $\begin{array}{l}674.94 \\
687.35 \\
692.09 \\
701.33 \\
737.73\end{array}$ & $\begin{array}{l}456.76 \\
455.38 \\
458.89 \\
458.93 \\
461.34\end{array}$ \\
\hline $\begin{array}{l}3099-47-18 B \\
399-1-1 \\
399-1-10 A \\
399-1-11 \\
399-1-12\end{array}$ & N & $\begin{array}{l}32.88 \\
34.96 \\
31.03 \\
34.92 \\
41.92\end{array}$ & $\begin{array}{l}374.95 \\
376.73 \\
373.65 \\
377.79 \\
384.45\end{array}$ & $\begin{array}{l}342.07 \\
341.77 \\
342.62 \\
342.87 \\
342.53\end{array}$ \\
\hline
\end{tabular}


Appendix A: June 1993 Water Level Measurement Data (Sheet 12 of 22)

\begin{tabular}{|c|c|c|c|c|}
\hline \multirow{2}{*}{ We11 } & \multirow{2}{*}{ Notes } & \multirow{2}{*}{$\begin{array}{l}\text { Depth to } \\
\text { Water, ft }\end{array}$} & \multicolumn{2}{|c|}{ Elevation, ft above msl } \\
\hline & & & Adjusted Casing & Water Level \\
\hline $\begin{array}{l}399-1-13 A \\
399-1-14 A \\
399-1-15 \\
399-1-16 A \\
399-1-16 B\end{array}$ & $\mathbf{N}$ & $\begin{array}{l}45.99 \\
40.24 \\
36.45 \\
39.41 \\
38.89\end{array}$ & $\begin{array}{l}388.62 \\
383.23 \\
379.59 \\
381.51 \\
381.14\end{array}$ & $\begin{array}{l}342.63 \\
342.99 \\
343.14 \\
342.10 \\
342.25\end{array}$ \\
\hline $\begin{array}{l}399-1-16 C \\
399-1-17 A \\
399-1-17 B \\
399-1-17 C \\
399-1-18 A\end{array}$ & $\begin{array}{l}N \\
N \\
N\end{array}$ & $\begin{array}{r}28.69 \\
35.09 \\
35.32 \\
1.99 \\
47.18\end{array}$ & $\begin{array}{l}382.29 \\
377.47 \\
377.87 \\
378.13 \\
390.83\end{array}$ & $\begin{array}{l}353.60 \\
342.38 \\
342.55 \\
376.14 \\
343.65\end{array}$ \\
\hline $\begin{array}{l}399-1-18 B \\
399-1-18 C \\
399-1-19 \\
399-1-2 \\
399-1-21 A\end{array}$ & $\begin{array}{l}N \\
N \\
N \\
N\end{array}$ & $\begin{array}{l}46.05 \\
43.60 \\
32.08 \\
47.15 \\
40.13\end{array}$ & $\begin{array}{l}389.94 \\
388.05 \\
374.64 \\
387.02 \\
382.39\end{array}$ & $\begin{array}{l}343.89 \\
344.45 \\
342.56 \\
339.87 \\
342.26\end{array}$ \\
\hline $\begin{array}{l}399-1-21 B \\
399-1-3 \\
399-1-4 \\
399-1-5 \\
399-1-6\end{array}$ & $N$ & $\begin{array}{l}41.04 \\
42.50 \\
37.66 \\
37.28 \\
30.88\end{array}$ & $\begin{array}{l}383.33 \\
384.73 \\
381.11 \\
379.82 \\
373.81\end{array}$ & $\begin{array}{l}342.29 \\
342.23 \\
343.45 \\
342.54 \\
342.93\end{array}$ \\
\hline $\begin{array}{l}399-1-7 \\
399-1-8 \\
399-1-9 \\
399-2-1 \\
399-2-3\end{array}$ & $\begin{array}{l}N \\
N \\
N\end{array}$ & $\begin{array}{l}43.44 \\
42.68 \\
26.15 \\
33.23 \\
33.39\end{array}$ & $\begin{array}{l}385.63 \\
384.91 \\
384.80 \\
375.30 \\
375.44\end{array}$ & $\begin{array}{l}342.19 \\
342.23 \\
358.65 \\
342.07 \\
342.05\end{array}$ \\
\hline $\begin{array}{l}399-3-1 \\
399-3-10 \\
399-3-6 \\
399-3-7 \\
399-3-9\end{array}$ & & $\begin{array}{l}42.44 \\
43.54 \\
50.48 \\
51.00 \\
46.19\end{array}$ & $\begin{array}{l}384.43 \\
385.40 \\
392.85 \\
394.22 \\
388.17\end{array}$ & $\begin{array}{l}341.99 \\
341.86 \\
342.37 \\
343.22 \\
341.98\end{array}$ \\
\hline $\begin{array}{l}399-4-1 \\
399-4-10 \\
399-4-11 \\
399-4-7 \\
399-4-9\end{array}$ & & $\begin{array}{l}53.79 \\
36.98 \\
62.49 \\
37.02 \\
40.41\end{array}$ & $\begin{array}{l}395.68 \\
377.59 \\
404.53 \\
378.64 \\
382.24\end{array}$ & $\begin{array}{l}341.89 \\
340.61 \\
342.01 \\
341.62 \\
341.83\end{array}$ \\
\hline
\end{tabular}


Appendix A: June 1993 Water Level Measurement Data (Sheet 13 of 22)

\begin{tabular}{|c|c|c|c|c|}
\hline \multirow{2}{*}{ Well } & \multirow{2}{*}{ Notes } & \multirow{2}{*}{$\begin{array}{l}\text { Depth to } \\
\text { Water, ft }\end{array}$} & \multicolumn{2}{|c|}{ Flevation, ft above msl } \\
\hline & & & Adjusted Casing & Water Level \\
\hline $\begin{array}{l}399-5-1 \\
399-6-1 \\
399-8-1 \\
399-8-2 \\
399-8-3\end{array}$ & $N$ & $\begin{array}{l}53.10 \\
48.07 \\
53.52 \\
56.56 \\
53.54\end{array}$ & $\begin{array}{l}395.60 \\
388.84 \\
396.20 \\
398.09 \\
394.82\end{array}$ & $\begin{array}{l}342.50 \\
340.77 \\
342.68 \\
341.53 \\
341.28\end{array}$ \\
\hline $\begin{array}{l}399-8-5 A \\
399-8-5 B \\
399-8-5 C \\
699-10-54 A \\
699-10-E 12\end{array}$ & $\begin{array}{l}N \\
N \\
N\end{array}$ & $\begin{array}{r}57.04 \\
56.02 \\
29.34 \\
102.99 \\
73.71\end{array}$ & $\begin{array}{l}400.31 \\
399.85 \\
399.91 \\
516.40 \\
430.86\end{array}$ & $\begin{array}{l}343.27 \\
343.83 \\
370.57 \\
413.41 \\
357.15\end{array}$ \\
\hline $\begin{array}{l}699-101-48 B \\
699-11-45 A \\
699-14-38 \\
699-14-47 \\
699-15-15 A\end{array}$ & & $\begin{array}{r}10.87 \\
166.65 \\
110.14 \\
175.22 \\
149.27\end{array}$ & $\begin{array}{l}390.15 \\
578.58 \\
514.89 \\
587.23 \\
547.14\end{array}$ & $\begin{array}{l}379.28 \\
411.93 \\
404.75 \\
412.01 \\
397.87\end{array}$ \\
\hline $\begin{array}{l}699-15-26 \\
699-17-5 \\
699-17-70 \\
699-19-43 \\
699-19-58\end{array}$ & $N$ & $\begin{array}{r}123.78 \\
45.47 \\
88.77 \\
148.45 \\
154.48\end{array}$ & $\begin{array}{l}523.83 \\
433.19 \\
563.18 \\
551.58 \\
573.05\end{array}$ & $\begin{array}{l}400.05 \\
387.72 \\
474.41 \\
403.13 \\
418.57\end{array}$ \\
\hline $\begin{array}{l}699-19-88 \\
699-2-3 \\
699-2-33 A \\
699-20-20 \\
699-20-39\end{array}$ & $\begin{array}{l}N \\
N \\
N \\
N\end{array}$ & $\begin{array}{r}130.76 \\
86.99 \\
131.99 \\
105.53 \\
138.12\end{array}$ & $\begin{array}{l}644.45 \\
477.14 \\
536.37 \\
505.58 \\
539.98\end{array}$ & $\begin{array}{l}513.69 \\
390.15 \\
404.38 \\
400.05 \\
401.86\end{array}$ \\
\hline $\begin{array}{l}699-20-E 12 \\
699-21-17 \\
699-23-34 \\
699-24-1 T \\
699-24-33\end{array}$ & $N$ & $\begin{array}{r}79.83 \\
131.06 \\
131.32 \\
101.95 \\
122.84\end{array}$ & $\begin{array}{l}437.25 \\
527.31 \\
532.84 \\
475.54 \\
524.27\end{array}$ & $\begin{array}{l}357.42 \\
396.25 \\
401.52 \\
373.59 \\
401.43\end{array}$ \\
\hline $\begin{array}{l}699-24-34 A \\
699-24-34 B \\
699-24-34 C \\
699-24-35 \\
699-25-33 A\end{array}$ & $N$ & $\begin{array}{l}132.36 \\
131.97 \\
131.08 \\
137.27 \\
127.04\end{array}$ & $\begin{array}{l}533.88 \\
533.50 \\
532.59 \\
538.81 \\
528.97\end{array}$ & $\begin{array}{l}401.52 \\
401.53 \\
401.51 \\
401.54 \\
401.93\end{array}$ \\
\hline
\end{tabular}


Appendix A: June 1993 Water Level Measurement Data

(Sheet 14 of 22)

\begin{tabular}{|c|c|c|c|c|}
\hline \multirow{2}{*}{ Well } & \multirow{2}{*}{ Notes } & \multirow{2}{*}{$\begin{array}{l}\text { Depth to } \\
\text { Water, ft }\end{array}$} & \multicolumn{2}{|c|}{ Elevation, ft above msl } \\
\hline & & & Adjusted Casing & Water Level \\
\hline $\begin{array}{l}699-25-34 A \\
699-25-34 B \\
699-25-34 C \\
699-25-34 D \\
699-25-55\end{array}$ & $\begin{array}{l}N \\
N \\
N\end{array}$ & $\begin{array}{l}128.32 \\
127.88 \\
133.94 \\
136.27 \\
264.09\end{array}$ & $\begin{array}{l}530.31 \\
529.40 \\
535.46 \\
537.91 \\
676.55\end{array}$ & $\begin{array}{l}401.99 \\
401.52 \\
401.52 \\
401.64 \\
412.46\end{array}$ \\
\hline $\begin{array}{l}699-25-70 \\
699-26-15 A \\
699-26-33 \\
699-26-34 \\
699-26-34 B\end{array}$ & $\begin{array}{l}N \\
N\end{array}$ & $\begin{array}{r}183.34 \\
45.19 \\
134.51 \\
126.87 \\
128.68\end{array}$ & $\begin{array}{l}629.56 \\
442.64 \\
535.76 \\
528.40 \\
530.27\end{array}$ & $\begin{array}{l}446.22 \\
397.45 \\
401.25 \\
401.53 \\
401.59\end{array}$ \\
\hline $\begin{array}{l}699-26-35 A \\
699-26-35 C \\
699-26-89 \\
699-27-8 \\
699-28-40\end{array}$ & $\begin{array}{l}N \\
N \\
N\end{array}$ & $\begin{array}{r}131.09 \\
131.12 \\
182.93 \\
72.54 \\
157.60\end{array}$ & $\begin{array}{l}532.66 \\
532.68 \\
653.08 \\
465.67 \\
559.44\end{array}$ & $\begin{array}{l}401.57 \\
401.56 \\
470.15 \\
393.13 \\
401.84\end{array}$ \\
\hline $\begin{array}{l}699-28-52 A \\
699-29-70 A P \\
699-29-70 A Q \\
699-29-70 C P \\
699-29-70 C Q\end{array}$ & $\begin{array}{l}N \\
P \\
P \\
P\end{array}$ & $\begin{array}{l}281.08 \\
191.03 \\
184.21 \\
229.90 \\
232.51\end{array}$ & $\begin{array}{l}684.67 \\
629.75 \\
630.15 \\
630.94 \\
630.99\end{array}$ & $\begin{array}{l}403.59 \\
438.72 \\
445.94 \\
401.04 \\
398.48\end{array}$ \\
\hline $\begin{array}{l}699-29-70 C R \\
699-29-70 C S \\
699-29-70 C T \\
699-29-70 C U \\
699-29-78\end{array}$ & $\begin{array}{l}p \\
p \\
P \\
P \\
N\end{array}$ & $\begin{array}{l}230.88 \\
233.34 \\
234.12 \\
235.20 \\
187.14\end{array}$ & $\begin{array}{l}631.15 \\
631.31 \\
631.48 \\
631.54 \\
647.05\end{array}$ & $\begin{array}{l}400.27 \\
397.97 \\
397.36 \\
396.44 \\
459.91\end{array}$ \\
\hline $\begin{array}{l}699-3-45 \\
699-31-31 \\
699-31-84 B \\
699-32-22 \\
699-32-43\end{array}$ & $N$ & $\begin{array}{r}92.48 \\
127.93 \\
204.49 \\
117.95 \\
114.67\end{array}$ & $\begin{array}{l}504.54 \\
529.32 \\
625.12 \\
517.55 \\
516.62\end{array}$ & $\begin{array}{l}412.06 \\
401.39 \\
420.63 \\
399.60 \\
401.95\end{array}$ \\
\hline $\begin{array}{l}699-32-62 \\
699-32-70 B \\
699-32-72 \\
699-32-77 \\
699-33-42\end{array}$ & $\begin{array}{l}N \\
N \\
N \\
N\end{array}$ & $\begin{array}{l}279.44 \\
217.63 \\
217.55 \\
195.96 \\
113.98\end{array}$ & $\begin{array}{l}707.09 \\
666.61 \\
668.16 \\
653.74 \\
516.00\end{array}$ & $\begin{array}{l}427.65 \\
448.98 \\
450.61 \\
457.78 \\
402.02\end{array}$ \\
\hline
\end{tabular}


WHC-EP-0394-7

Appendix A: June 1993 Water Level Measurement Data

(Sheet 15 of 22)

\begin{tabular}{|c|c|c|c|c|}
\hline \multirow{2}{*}{ Well } & \multirow{2}{*}{ Notes } & \multirow{2}{*}{$\begin{array}{l}\text { Depth to } \\
\text { Water, ft }\end{array}$} & \multicolumn{2}{|c|}{ Elevation, ft above ms 1} \\
\hline & & & Adjusted Casing & Water Level \\
\hline $\begin{array}{l}699-33-56 \\
699-34-39 A \\
699-34-41 B \\
699-34-42 \\
699-34-51\end{array}$ & $\begin{array}{l}N \\
N \\
N\end{array}$ & $\begin{array}{l}313.99 \\
135.17 \\
168.90 \\
138.18 \\
334.43\end{array}$ & $\begin{array}{l}717.03 \\
537.07 \\
570.89 \\
540.20 \\
736.76\end{array}$ & $\begin{array}{l}403.04 \\
401.90 \\
401.99 \\
402.02 \\
402.33\end{array}$ \\
\hline $\begin{array}{l}699-34-88 \\
699-35-66 \\
699-35-70 \\
699-35-78 A \\
699-35-9\end{array}$ & & $\begin{array}{l}164.57 \\
288.22 \\
244.99 \\
198.33 \\
115.06\end{array}$ & $\begin{array}{l}632.82 \\
725.65 \\
693.72 \\
660.65 \\
499.83\end{array}$ & $\begin{array}{l}468.25 \\
437.43 \\
448.73 \\
462.32 \\
384.77\end{array}$ \\
\hline $\begin{array}{l}699-36-61 A \\
699-36-93 \\
699-37-43 \\
699-37-82 A \\
699-38-65\end{array}$ & $N$ & $\begin{array}{l}341.66 \\
174.31 \\
287.54 \\
172.33 \\
324.14\end{array}$ & $\begin{array}{l}748.11 \\
644.77 \\
690.58 \\
636.75 \\
753.33\end{array}$ & $\begin{array}{l}406.45 \\
470.46 \\
403.04 \\
464.42 \\
429.19\end{array}$ \\
\hline $\begin{array}{l}699-38-70 \\
699-39-39 \\
699-39-79 \\
699-40-1 \\
699-40-33 A\end{array}$ & & $\begin{array}{r}260.65 \\
124.55 \\
210.18 \\
75.55 \\
107.82\end{array}$ & $\begin{array}{l}710.67 \\
536.65 \\
673.52 \\
438.71 \\
518.05\end{array}$ & $\begin{array}{l}450.02 \\
412.10 \\
463.34 \\
363.16 \\
410.23\end{array}$ \\
\hline $\begin{array}{l}699-40-36 \\
699-40-39 \\
699-40-40 A \\
699-40-40 B \\
699-40-62\end{array}$ & $\begin{array}{l}N \\
N \\
N\end{array}$ & $\begin{array}{l}117.86 \\
129.27 \\
129.80 \\
130.48 \\
342.84\end{array}$ & $\begin{array}{l}528.92 \\
541.84 \\
541.21 \\
542.18 \\
747.78\end{array}$ & $\begin{array}{l}411.06 \\
412.57 \\
411.41 \\
411.70 \\
404.94\end{array}$ \\
\hline $\begin{array}{l}699-41-23 \\
699-41-35 \\
699-41-40 \\
699-42-12 A \\
699-42-37\end{array}$ & $N$ & $\begin{array}{r}69.54 \\
108.27 \\
130.52 \\
139.19 \\
104.42\end{array}$ & $\begin{array}{l}466.50 \\
520.38 \\
545.94 \\
514.27 \\
519.42\end{array}$ & $\begin{array}{l}396.96 \\
412.11 \\
415.42 \\
375.08 \\
415.00\end{array}$ \\
\hline $\begin{array}{l}699-42-39 A \\
699-42-39 B \\
699-42-40 A \\
699-42-40 B \\
699-42-40 C\end{array}$ & $\begin{array}{l}N \\
N \\
N \\
P\end{array}$ & $\begin{array}{l}139.15 \\
139.57 \\
124.23 \\
124.74 \\
133.24\end{array}$ & $\begin{array}{l}558.14 \\
558.32 \\
545.43 \\
546.46 \\
546.16\end{array}$ & $\begin{array}{l}418.99 \\
418.75 \\
421.20 \\
421.72 \\
412.92\end{array}$ \\
\hline
\end{tabular}


Appendix A: June 1993 Water Level Measurement Data

(Sheet 16 of 22)

\begin{tabular}{|c|c|c|c|c|}
\hline \multirow{2}{*}{ Well } & \multirow{2}{*}{ Notes } & \multirow{2}{*}{$\begin{array}{l}\text { Depth to } \\
\text { Water, ft }\end{array}$} & \multicolumn{2}{|c|}{ Elevation, ft above $\mathrm{msl}$} \\
\hline & & & Adjusted Casing & Water Level \\
\hline $\begin{array}{l}699-42-41 \\
699-42-42 B \\
699-43-104 \\
699-43-40 \\
699-43-41 E\end{array}$ & $N$ & $\begin{array}{l}146.92 \\
166.34 \\
271.15 \\
122.46 \\
130.20\end{array}$ & $\begin{array}{l}567.30 \\
583.23 \\
766.07 \\
542.20 \\
550.86\end{array}$ & $\begin{array}{l}420.38 \\
416.89 \\
494.92 \\
419.74 \\
420.66\end{array}$ \\
\hline $\begin{array}{l}699-43-41 F \\
699-43-41 G \\
699-43-42 \\
699-43-42 J \\
699-43-43\end{array}$ & $N$ & $\begin{array}{l}130.28 \\
135.05 \\
143.71 \\
163.02 \\
164.39\end{array}$ & $\begin{array}{l}551.01 \\
551.34 \\
564.48 \\
581.68 \\
579.37\end{array}$ & $\begin{array}{l}420.73 \\
416.29 \\
420.77 \\
418.66 \\
414.98\end{array}$ \\
\hline $\begin{array}{l}699-43-45 \\
699-43-89 \\
699-43-91 A P \\
699-43-91 A Q \\
699-43-910\end{array}$ & $\begin{array}{l}N \\
N \\
N\end{array}$ & $\begin{array}{l}194.44 \\
178.70 \\
231.40 \\
231.43 \\
261.79\end{array}$ & $\begin{array}{l}597.68 \\
644.15 \\
671.51 \\
671.94 \\
671.99\end{array}$ & $\begin{array}{l}403.24 \\
465.45 \\
440.11 \\
440.51 \\
410.20\end{array}$ \\
\hline $\begin{array}{l}699-44-42 \\
699-44-43 B \\
699-44-64 \\
699-44-91 P \\
699-44-91 Q\end{array}$ & $\begin{array}{l}N \\
N\end{array}$ & $\begin{array}{l}158.56 \\
164.64 \\
319.93 \\
267.94 \\
268.17\end{array}$ & $\begin{array}{l}579.22 \\
580.12 \\
725.60 \\
672.15 \\
672.33\end{array}$ & $\begin{array}{l}420.66 \\
415.48 \\
405.67 \\
404.21 \\
404.16\end{array}$ \\
\hline $\begin{array}{l}699-44-91 R \\
699-44-91 S \\
699-44-91 T \\
699-44-91 U \\
699-45-42\end{array}$ & $\begin{array}{l}N \\
N \\
N \\
N\end{array}$ & $\begin{array}{l}266.85 \\
274.05 \\
274.92 \\
275.24 \\
161.40\end{array}$ & $\begin{array}{l}672.49 \\
672.68 \\
672.84 \\
673.01 \\
577.33\end{array}$ & $\begin{array}{l}405.64 \\
398.63 \\
397.92 \\
397.77 \\
415.93\end{array}$ \\
\hline $\begin{array}{l}699-46-21 B \\
699-47-35 A \\
699-47-35 B \\
699-47-46 A \\
699-47-50\end{array}$ & $\begin{array}{l}N \\
N\end{array}$ & $\begin{array}{r}131.73 \\
63.40 \\
63.71 \\
176.99 \\
180.94\end{array}$ & $\begin{array}{l}522.02 \\
476.36 \\
476.65 \\
580.14 \\
584.22\end{array}$ & $\begin{array}{l}390.29 \\
412.96 \\
412.94 \\
403.15 \\
403.28\end{array}$ \\
\hline $\begin{array}{l}699-47-60 \\
699-47-80 A P \\
699-47-80 A Q \\
699-47-80 C P \\
699-47-80 C Q\end{array}$ & $\begin{array}{l}N \\
N \\
N \\
N\end{array}$ & $\begin{array}{l}249.70 \\
272.04 \\
262.35 \\
307.74 \\
308.75\end{array}$ & $\begin{array}{l}651.52 \\
713.03 \\
713.51 \\
712.58 \\
712.75\end{array}$ & $\begin{array}{l}401.82 \\
440.99 \\
451.16 \\
404.84 \\
404.00\end{array}$ \\
\hline
\end{tabular}


Appendix A: June 1993 Water Level Measurement Data

(Sheet 17 of 22)

\begin{tabular}{|c|c|c|c|c|}
\hline \multirow{2}{*}{ Well } & \multirow{2}{*}{ Notes } & \multirow{2}{*}{$\begin{array}{l}\text { Depth to } \\
\text { Water, ft }\end{array}$} & \multicolumn{2}{|c|}{ Elevation, ft above msl } \\
\hline & & & Adjusted Casing & Water Level \\
\hline $\begin{array}{l}699-47-80 C R \\
699-47-80 C S \\
699-47-80 C T \\
699-47-80 C U \\
699-48-7\end{array}$ & $\begin{array}{l}N \\
N \\
N \\
N \\
N\end{array}$ & $\begin{array}{r}308.70 \\
314.28 \\
314.62 \\
314.79 \\
26.50\end{array}$ & $\begin{array}{l}712.92 \\
713.10 \\
713.25 \\
713.43 \\
384.72\end{array}$ & $\begin{array}{l}404.22 \\
398.82 \\
398.63 \\
398.64 \\
358.22\end{array}$ \\
\hline $\begin{array}{l}699-48-71 \\
699-49-13 E \\
699-49=28 \\
699-49-55 A \\
699-49-55 B\end{array}$ & $P$ & $\begin{array}{r}243.82 \\
51.55 \\
141.79 \\
129.08 \\
129.12\end{array}$ & $\begin{array}{l}688.15 \\
412.72 \\
535.40 \\
531.03 \\
531.12\end{array}$ & $\begin{array}{l}444.33 \\
361.17 \\
393.61 \\
401.95 \\
402.00\end{array}$ \\
\hline $\begin{array}{l}699-49-79 \\
699-50-28 B \\
699-50-30 \\
699-50-42 \\
699-50-45\end{array}$ & $P$ & $\begin{array}{r}233.47 \\
143.70 \\
134.82 \\
56.47 \\
43.35\end{array}$ & $\begin{array}{l}688.59 \\
537.30 \\
528.84 \\
466.84 \\
451.41\end{array}$ & $\begin{array}{l}455.12 \\
393.60 \\
394.02 \\
410.37 \\
408.06\end{array}$ \\
\hline $\begin{array}{l}699-50-48 B \\
699-50-85 \\
699-51-46 \\
699-51-63 \\
699-51-75\end{array}$ & $\begin{array}{l}N \\
P\end{array}$ & $\begin{array}{r}145.00 \\
284.93 \\
38.27 \\
167.63 \\
193.20\end{array}$ & $\begin{array}{l}550.39 \\
739.35 \\
444.63 \\
571.84 \\
641.51\end{array}$ & $\begin{array}{l}405.39 \\
454.42 \\
406.36 \\
404.21 \\
448.31\end{array}$ \\
\hline $\begin{array}{l}699-52-19 \\
699-52-46 A \\
699-52-48 \\
699-52-54 \\
699-52-57\end{array}$ & $\begin{array}{l}P \\
P \\
N \\
N\end{array}$ & $\begin{array}{r}49.70 \\
47.43 \\
61.95 \\
166.75 \\
157.14\end{array}$ & $\begin{array}{l}411.08 \\
455.61 \\
466.06 \\
568.29 \\
558.46\end{array}$ & $\begin{array}{l}361.38 \\
408.18 \\
404.11 \\
401.54 \\
401.32\end{array}$ \\
\hline $\begin{array}{l}699-53-35 \\
699-53-47 A \\
699-53-47 B \\
599-53-48 A \\
699-53-48 B\end{array}$ & $\begin{array}{l}N \\
N \\
N\end{array}$ & $\begin{array}{r}130.92 \\
32.69 \\
32.98 \\
39.81 \\
38.07\end{array}$ & $\begin{array}{l}530.99 \\
438.28 \\
438.58 \\
442.85 \\
442.71\end{array}$ & $\begin{array}{l}400.07 \\
405.59 \\
405.60 \\
403.04 \\
404.64\end{array}$ \\
\hline $\begin{array}{l}699-53-50 \\
699-53-55 A \\
699-53-55 B \\
69{ }^{\circ}-53-55 C \\
699-54-19\end{array}$ & $\begin{array}{l}P \\
N \\
N\end{array}$ & $\begin{array}{r}40.63 \\
175.27 \\
175.86 \\
174.84 \\
22.18\end{array}$ & $\begin{array}{l}444.21 \\
576.56 \\
576.84 \\
576.13 \\
383.60\end{array}$ & $\begin{array}{l}403.58 \\
401.29 \\
400.98 \\
401.29 \\
361.42\end{array}$ \\
\hline
\end{tabular}


Appendix A: June 1993 Water Level Measurement Data

(Sheet 18 of 22)

\begin{tabular}{|c|c|c|c|c|}
\hline \multirow{2}{*}{ Well } & \multirow{2}{*}{ Notes } & \multirow{2}{*}{$\begin{array}{l}\text { Depth to } \\
\text { Water, ft }\end{array}$} & \multicolumn{2}{|c|}{ Elevation, ft above msl } \\
\hline & & & Adjusted Casing & Water Level \\
\hline $\begin{array}{l}699-54-34 \\
699-54-37 A \\
699-54-42 \\
699-54-45 A \\
699-54-48\end{array}$ & $\begin{array}{l}Q \\
Q \\
Q \\
Q\end{array}$ & $\begin{array}{r}140.47 \\
124.25 \\
115.33 \\
96.56 \\
55.77\end{array}$ & $\begin{array}{l}550.24 \\
534.17 \\
511.49 \\
494.29 \\
457.02\end{array}$ & $\begin{array}{l}409.77 \\
409.92 \\
396.16 \\
397.73 \\
401.25\end{array}$ \\
\hline $\begin{array}{l}699-54-57 \\
699-55-21 \\
699-55-40 \\
699-55-44 \\
699-55-50 C\end{array}$ & $\begin{array}{l}P \\
N \\
Q \\
Q\end{array}$ & $\begin{array}{r}174.81 \\
35.77 \\
134.12 \\
123.94 \\
43.19\end{array}$ & $\begin{array}{l}576.24 \\
395.96 \\
543.13 \\
519.67 \\
444.43\end{array}$ & $\begin{array}{l}401.43 \\
360.19 \\
409.01 \\
395.73 \\
401.24\end{array}$ \\
\hline $\begin{array}{l}699-55-55 \\
699-55-57 \\
699-55-70 \\
699-55-76 \\
699-55-89\end{array}$ & $N$ & $\begin{array}{l}162.82 \\
166.94 \\
137.64 \\
139.92 \\
163.16\end{array}$ & $\begin{array}{l}563.76 \\
568.22 \\
569.03 \\
583.24 \\
617.43\end{array}$ & $\begin{array}{l}400.94 \\
401.28 \\
431.39 \\
443.32 \\
454.27\end{array}$ \\
\hline $\begin{array}{l}699-55-95 \\
699-56-43 \\
699-56-53 \\
699-57-25 A \\
699-57-29 A\end{array}$ & $\begin{array}{l}N \\
P \\
P \\
N \\
N\end{array}$ & $\begin{array}{r}312.05 \\
132.27 \\
32.74 \\
50.87 \\
54.30\end{array}$ & $\begin{array}{l}777.05 \\
540.42 \\
434.34 \\
414.57 \\
415.44\end{array}$ & $\begin{array}{l}465.00 \\
408.15 \\
401.60 \\
363.70 \\
361.14\end{array}$ \\
\hline $\begin{array}{l}699-57-29 B \\
699-57-83 A \\
699-58-24 \\
699-59-32 \\
699-59-58\end{array}$ & $\begin{array}{l}N \\
N\end{array}$ & $\begin{array}{r}54.76 \\
145.82 \\
57.45 \\
62.67 \\
97.01\end{array}$ & $\begin{array}{l}416.18 \\
577.96 \\
418.80 \\
424.29 \\
498.02\end{array}$ & $\begin{array}{l}361.42 \\
432.14 \\
361.35 \\
361.62 \\
401.01\end{array}$ \\
\hline $\begin{array}{l}699-59-80 B \\
699-60-32 \\
699-60-57 \\
699-60-60 \\
699-61-37\end{array}$ & $\begin{array}{l}N \\
N\end{array}$ & $\begin{array}{r}153.78 \\
63.85 \\
68.19 \\
110.98 \\
61.56\end{array}$ & $\begin{array}{l}583.25 \\
425.30 \\
469.57 \\
512.04 \\
442.94\end{array}$ & $\begin{array}{l}429.47 \\
361.45 \\
401.38 \\
401.06 \\
381.38\end{array}$ \\
\hline $\begin{array}{l}699-61-41 \\
699-61-62 \\
699-61-66 \\
699-62-31 \\
699-62-43 A\end{array}$ & $N$ & $\begin{array}{r}33.30 \\
96.45 \\
121.96 \\
72.71 \\
36.40\end{array}$ & $\begin{array}{l}428.92 \\
497.51 \\
522.18 \\
434.12 \\
432.30\end{array}$ & $\begin{array}{l}395.62 \\
401.06 \\
400.22 \\
361.41 \\
395.90\end{array}$ \\
\hline
\end{tabular}


Appendix A: June 1993 Water Leve1 Measurement Data

(Sheet 19 of 22)

\begin{tabular}{|c|c|c|c|c|}
\hline \multirow{2}{*}{ Well } & \multirow{2}{*}{ Notes } & \multirow{2}{*}{$\begin{array}{l}\text { Depth to } \\
\text { Water, ft }\end{array}$} & \multicolumn{2}{|c|}{ Elevation, ft above msl } \\
\hline & & & Adjusted Casing & Water Level \\
\hline $\begin{array}{l}699-63-25 A \\
699-63-51 \\
699-63-55 \\
699-63-58 \\
699-63-90\end{array}$ & & $\begin{array}{r}33.86 \\
25.53 \\
26.92 \\
91.94 \\
110.43\end{array}$ & $\begin{array}{l}395.15 \\
424.54 \\
426.54 \\
491.90 \\
509.73\end{array}$ & $\begin{array}{l}361.29 \\
399.01 \\
399.62 \\
399.96 \\
399.30\end{array}$ \\
\hline $\begin{array}{l}699-63-92 \\
699-64-27 \\
699-64-62 \\
699-65-22 \\
699-65-23\end{array}$ & $\begin{array}{l}N \\
N \\
N\end{array}$ & $\begin{array}{r}83.56 \\
52.95 \\
100.53 \\
29.47 \\
25.86\end{array}$ & $\begin{array}{l}484.20 \\
414.29 \\
500.25 \\
391.10 \\
387.93\end{array}$ & $\begin{array}{l}400.64 \\
361.34 \\
399.72 \\
361.63 \\
362.07\end{array}$ \\
\hline $\begin{array}{l}699-65-50 \\
699-65-59 A \\
699-65-72 \\
699-65-83 \\
699-65-95\end{array}$ & $P$ & $\begin{array}{r}68.09 \\
107.36 \\
142.24 \\
87.99 \\
51.72\end{array}$ & $\begin{array}{l}467.06 \\
506.96 \\
540.28 \\
485.63 \\
452.26\end{array}$ & $\begin{array}{l}398.97 \\
399.60 \\
398.04 \\
397.64 \\
400.54\end{array}$ \\
\hline $\begin{array}{l}699-66-103 \\
699-66-23 \\
699-66-38 \\
699-66-39 \\
699-66-58\end{array}$ & $N$ & $\begin{array}{r}62.18 \\
26.42 \\
34.01 \\
47.15 \\
103.79\end{array}$ & $\begin{array}{l}463.01 \\
389.01 \\
436.24 \\
453.78 \\
503.33\end{array}$ & $\begin{array}{l}400.83 \\
362.59 \\
402.23 \\
406.63 \\
399.54\end{array}$ \\
\hline $\begin{array}{l}699-66-64 \\
699-66-91 \\
699-67-51 \\
699-67-86 \\
699-67-98\end{array}$ & $N$ & $\begin{array}{r}106.83 \\
68.43 \\
125.52 \\
74.84 \\
54.77\end{array}$ & $\begin{array}{l}505.92 \\
467.75 \\
524.59 \\
472.39 \\
455.47\end{array}$ & $\begin{array}{l}399.09 \\
399.32 \\
399.07 \\
397.55 \\
400.70\end{array}$ \\
\hline $\begin{array}{l}699-68-105 \\
699-69-38 \\
699-69-450 \\
699-70-23 \\
699-70-68\end{array}$ & $Q$ & $\begin{array}{r}62.47 \\
21.04 \\
88.58 \\
28.71 \\
128.06\end{array}$ & $\begin{array}{l}451.85 \\
423.52 \\
487.18 \\
391.71 \\
526.21\end{array}$ & $\begin{array}{l}389.38 \\
402.48 \\
398.60 \\
363.00 \\
398.15\end{array}$ \\
\hline $\begin{array}{l}699-71-30 \\
699-71-52 \\
699-71-77 \\
699-72-73 \\
699-72-88\end{array}$ & & $\begin{array}{r}30.39 \\
124.59 \\
76.21 \\
86.20 \\
36.41\end{array}$ & $\begin{array}{l}400.68 \\
523.04 \\
472.28 \\
482.57 \\
437.37\end{array}$ & $\begin{array}{l}370.29 \\
398.45 \\
396.07 \\
396.37 \\
400.96\end{array}$ \\
\hline
\end{tabular}


Appendix A: June 1993 Water Level Measurement Data (Sheet 20 of 22)

\begin{tabular}{|c|c|c|c|c|}
\hline \multirow{2}{*}{ Well } & \multirow{2}{*}{ Notes } & \multirow{2}{*}{$\begin{array}{l}\text { Depth to } \\
\text { Water, ft }\end{array}$} & \multicolumn{2}{|c|}{ Elevation, ft above msl } \\
\hline & & & Adjusted Casing & Water Level \\
\hline $\begin{array}{l}699-72-92 \\
699-72-98 \\
699-73-61 \\
699-74-44 \\
699-74-48\end{array}$ & & $\begin{array}{r}51.99 \\
50.09 \\
133.30 \\
48.80 \\
89.82\end{array}$ & $\begin{array}{l}452.22 \\
454.19 \\
531.53 \\
445.18 \\
487.18\end{array}$ & $\begin{array}{l}400.23 \\
404.10 \\
398.23 \\
396.38 \\
397.36\end{array}$ \\
\hline $\begin{array}{l}699-77-36 \\
699-77-54 \\
699-78-62 \\
699-8-17 \\
699-8-25\end{array}$ & & $\begin{array}{r}36.53 \\
84.03 \\
75.41 \\
124.76 \\
110.39\end{array}$ & $\begin{array}{l}412.28 \\
480.59 \\
469.88 \\
522.44 \\
509.30\end{array}$ & $\begin{array}{l}375.75 \\
396.56 \\
394.47 \\
397.68 \\
398.91\end{array}$ \\
\hline $\begin{array}{l}699-8-32 \\
699-80-43 S \\
699-81-38 \\
699-81-58 \\
699-82-45 A\end{array}$ & N & $\begin{array}{r}154.87 \\
24.93 \\
27.41 \\
46.66 \\
25.06\end{array}$ & $\begin{array}{l}554.39 \\
412.52 \\
406.47 \\
439.55 \\
413.73\end{array}$ & $\begin{array}{l}399.52 \\
387.59 \\
379.06 \\
392.89 \\
388.67\end{array}$ \\
\hline $\begin{array}{l}699-83-36 \\
699-83-47 \\
699-84-35 A \\
699-86-42 \\
699-87-42 A\end{array}$ & $Q$ & $\begin{array}{r}41.03 \\
46.79 \\
7.07 \\
25.41 \\
32.71\end{array}$ & $\begin{array}{l}418.63 \\
435.27 \\
400.28 \\
409.92 \\
416.53\end{array}$ & $\begin{array}{l}377.60 \\
388.48 \\
393.21 \\
384.51 \\
383.82\end{array}$ \\
\hline $\begin{array}{l}699-87-55 \\
699-88-41 \\
699-89-35 \\
699-9-E 2 \\
699-90-34\end{array}$ & N & $\begin{array}{l}72.20 \\
33.90 \\
26.34 \\
45.83 \\
20.90\end{array}$ & $\begin{array}{l}458.63 \\
416.04 \\
397.46 \\
418.09 \\
392.39\end{array}$ & $\begin{array}{l}386.43 \\
382.14 \\
371.12 \\
372.26 \\
371.49\end{array}$ \\
\hline $\begin{array}{l}699-90-45 \\
699-91-37 \\
699-91-46 \\
699-92-49 \\
699-93-48\end{array}$ & $\begin{array}{l}N \\
N\end{array}$ & $\begin{array}{l}37.68 \\
48.98 \\
33.21 \\
48.75 \\
54.97\end{array}$ & $\begin{array}{l}421.60 \\
422.93 \\
417.06 \\
431.94 \\
437.79\end{array}$ & $\begin{array}{l}383.92 \\
373.95 \\
383.85 \\
383.19 \\
382.82\end{array}$ \\
\hline $\begin{array}{l}699-96-43 \\
699-96-49 \\
699-97-43 \\
699-97-51 A \\
699-98-49 A\end{array}$ & N & $\begin{array}{l}42.80 \\
37.24 \\
43.06 \\
20.25 \\
19.94\end{array}$ & $\begin{array}{l}421.84 \\
419.26 \\
421.84 \\
402.33 \\
401.80\end{array}$ & $\begin{array}{l}379.04 \\
382.02 \\
378.78 \\
382.08 \\
381.86\end{array}$ \\
\hline
\end{tabular}


Appendix A: June 1993 Water Level Measurement Data

(Sheet 21 of 22)

\begin{tabular}{|c|c|c|c|c|}
\hline \multirow{2}{*}{ We11 } & \multirow{2}{*}{ Notes } & \multirow{2}{*}{$\begin{array}{l}\text { Depth to } \\
\text { Water, ft }\end{array}$} & \multicolumn{2}{|c|}{ Elevation, ft above msl } \\
\hline & & & Adjusted Casing & Water Level \\
\hline $\begin{array}{l}699-S 12-29 \\
699-S 12-3 \\
699-S 14-20 A \\
699-S 18-E 2 A \\
699-S 19-11\end{array}$ & $N$ & $\begin{array}{l}83.38 \\
54.75 \\
92.01 \\
75.47 \\
93.78\end{array}$ & $\begin{array}{l}487.68 \\
435.52 \\
492.74 \\
434.85 \\
483.74\end{array}$ & $\begin{array}{l}404.30 \\
380.77 \\
400.73 \\
359.38 \\
389.96\end{array}$ \\
\hline $\begin{array}{l}699-S 19-E 13 \\
699-S 19-E 14 \\
699-S 22-E 9 A \\
699-S 22-E 9 B \\
699-S 27-E 14\end{array}$ & $N$ & $\begin{array}{l}50.29 \\
30.06 \\
29.05 \\
27.96 \\
57.89\end{array}$ & $\begin{array}{l}394.51 \\
373.86 \\
374.19 \\
373.71 \\
399.76\end{array}$ & $\begin{array}{l}344.22 \\
343.80 \\
345.14 \\
345.75 \\
341.87\end{array}$ \\
\hline $\begin{array}{l}699-S 27-E 9 A \\
699-S 27-E 9 B \\
699-S 27-E 9 C \\
699-S 28-E 12 \\
699-S 29-E 16 A\end{array}$ & $\begin{array}{l}N \\
N\end{array}$ & $\begin{array}{r}42.66 \\
41.14 \\
6.20 \\
45.21 \\
38.34\end{array}$ & $\begin{array}{l}390.26 \\
390.42 \\
390.56 \\
389.76 \\
379.73\end{array}$ & $\begin{array}{l}347.60 \\
349.28 \\
384.36 \\
344.55 \\
341.39\end{array}$ \\
\hline $\begin{array}{l}699-S 29-E 16 B \\
699-S 29-E 16 C \\
699-S 3-25 \\
699-S 3-E 12 \\
699-S 30-E 10 A\end{array}$ & $\begin{array}{l}N \\
N\end{array}$ & $\begin{array}{r}38.40 \\
3.45 \\
124.75 \\
43.65 \\
43.78\end{array}$ & $\begin{array}{l}379.88 \\
379.48 \\
523.50 \\
397.90 \\
392.29\end{array}$ & $\begin{array}{l}341.48 \\
376.03 \\
398.75 \\
354.25 \\
348.51\end{array}$ \\
\hline $\begin{array}{l}699-S 30-E 10 B \\
699-S 30-E 15 A \\
699-S 31-1 \\
699-S 31-E 10 A \\
699-S 31-E 10 B\end{array}$ & & $\begin{array}{l}43.03 \\
57.77 \\
81.49 \\
36.59 \\
34.95\end{array}$ & $\begin{array}{l}392.07 \\
400.14 \\
460.00 \\
384.57 \\
383.71\end{array}$ & $\begin{array}{l}349.04 \\
342.37 \\
378.51 \\
347.98 \\
348.76\end{array}$ \\
\hline $\begin{array}{l}699-S 31-E 10 C \\
699-S 31-E 100 \\
699-S 31-E 8 \\
699-S 32-E 13 A \\
699-S 32-E 13 B\end{array}$ & N & $\begin{array}{l}34.27 \\
32.03 \\
20.82 \\
43.58 \\
47.75\end{array}$ & $\begin{array}{l}382.91 \\
380.58 \\
374.75 \\
390.46 \\
394.72\end{array}$ & $\begin{array}{l}348.64 \\
348.55 \\
353.93 \\
346.88 \\
346.97\end{array}$ \\
\hline $\begin{array}{l}699-S 32-E 8 \\
699-S 34-E 10 \\
699-S 34-E 15 \\
699-S 36-E 12 B \\
699-S 36-E 13 A\end{array}$ & N & $\begin{array}{l}15.53 \\
29.48 \\
52.31 \\
42.98 \\
43.66\end{array}$ & $\begin{array}{l}375.50 \\
382.37 \\
404.55 \\
399.07 \\
399.30\end{array}$ & $\begin{array}{l}359.97 \\
352.89 \\
352.24 \\
356.09 \\
355.64\end{array}$ \\
\hline
\end{tabular}


Appendix A: June 1993 Water Level Measurement Data (Sheet 22 of 22)

\begin{tabular}{c|c|c|c|c}
\hline \multirow{2}{*}{ We11 } & \multirow{2}{*}{ Notes } & $\begin{array}{c}\text { Depth to } \\
\text { Water, ft }\end{array}$ & \multicolumn{2}{|c}{ Elevation, ft above ms 1 } \\
\cline { 3 - 5 } & & & Adjusted Casing & Water Leve1 \\
\hline $699-S 37-E 11$ & & 43.77 & 399.30 & 355.53 \\
$699-S 37-E 14$ & & 51.46 & 408.28 & 356.82 \\
$699-S 38-E 11$ & $N$ & 43.86 & 398.60 & 354.74 \\
$699-S 38-E 12 A$ & & 47.84 & 404.95 & 357.11 \\
$699-S 38-E 12 B$ & $N$ & 47.84 & 405.00 & 357.16 \\
& & 41.05 & 402.85 & 361.80 \\
$699-S 40-E 14$ & & 46.95 & 401.36 & 354.41 \\
$699-S 41-E 11$ & & 46.78 & 401.93 & 355.15 \\
$699-S 41-E 12$ & & 51.27 & 410.56 & 359.29 \\
$699-S 41-E 13 A$ & & 50.72 & 410.10 & 359.38 \\
$699-S 41-E 13 B$ & $N$ & & & \\
& & 51.65 & 410.67 & 359.02 \\
$699-S 41-E 13 C$ & $N$ & 52.01 & 405.69 & 353.68 \\
$699-S 43-E 12$ & & 27.92 & 378.29 & 350.37 \\
$699-S 6-E 14 A$ & & 58.04 & 430.47 & 372.43 \\
$699-S 6-E 40$ & & 118.83 & 527.12 & 408.29 \\
$699-S 7-34$ & & 107.23 & 503.81 & 396.58 \\
$699-S 8-19$ & & & &
\end{tabular}

Notes: $N=$ Well not included on map

$P=$ Well in confined aquifer, not included on map

$Q=$ Questionable data, not included on map 
WHC-EP-0394-7

DISTRIBUTION

Number of copies

Offsite

1

U.S. Geological Survey

B. W. Drost

1201 Pacific Ave.

Suite 600

Tacoma, WA 98402

Onsite

4

U.S. Department of Energy, Richland Operations office

G. J. Bracken

R3-81

M. J. Furman

R3-80

A. J. Lassila

R3-73

K. M. Thompson

A5-15

2

Washington St te Department of Ecology

S. Leja

B5-18

M. Selby

B5-18

2

U.S. Environmental Protection Agency

D. R. Sherwood (2)

B5-01

12

Pacific Northwest Laboratory

M. P. Bergeron

K6-77

J. V. Borghese

K6-96

R. W. Bryce

M. A. Chamness

K6-96

K6-84

M. D. Freshley

K6-77

G. V. Last

E. W. Lusty

S. P. Luttrell

K6-84

$\mathrm{K} 6-79$

D. R. Newcomer

K6-96

R. M. Smith

F. A. Spane

K6-96

K6-96

K6-96

W. D. Webber

K6-96

68

Westinghouse Hanford Company

M. R. Adams

D. J. Alexander

D. B. Barnett

M. A. Buckmaster

H6-01

H6-06

H6-06

H6- 03

Distr-1 
Westinghouse Hanford Company (cont)

J. A. Caggiano

H6-06

L. B. Collard

H6-01

J. D. Davis

L. P. Diediker

J. J. Dorian

G. L. Dunford

H6-01

R. S. Edrington

TI -30

D. B. Erb

J. W. Fassett

K. R. Fecht

M. A. Frank

B. H. Ford

M. J. Hartman

F. D. Hodges

D. G. Horton

R. L. Jackson

V. G. Johnson

W. L. Johnson

W. A. Jordan

G. L. Kasza (5)

A. J. Knepp

M. J. Lauterbach

A. G. Law

J. W. Lindberg

K. A. Lindsey

H6-30

$\mathrm{R} 1-51$

H6-06

H6-03

H6-06

H6-06

H6-03

H6-06

H6-06

H6-06

H6-06

H6-06

H6-06

H6-04

H6-06

H6-06

H6-06

H6-01

H6-06

H6-06

A. H. Lu

C. J. Lynch

H6-06

HO-36

W. J. McMahon

H6-07

R. B. Mercer

H6-06

R. D. Miller

H6-06

D. J. Moak

S. E. Myers

R. S. Paul ina

H6-06

N3-05

N3-06

R3-45

R. E. Peterson

H6-06

S. M. Price

H6-23

W. H. Price

N3-C5

R. F. Raidl

S. P. Reidel

K. D. Reynolds

V. J. Rohay

A. L. Schatz

J. S. Schmid

J. A. Serkowski

H6-06

H6-06

H6-06

H6-06

N3-05

H6-06

H6-06

K. R. Simpson

H6-06

L. C. Swanson

H6-06

M. D. Sweeney

H6-06

K. J. Swett

H6-06

R. R. Thompson

H6-32

E. C. Thornton

H6-06

S. J. Trent

H6-06

P. J. Valcich

H6-04

D. C. Weekes

H6-06 
WHC-EP-0394-7

Westinghouse Hanford Company (cont)

C. D. Wittreich

Central Files (2)

H6-03

EPIC (2)

IRA (3)

Public Reading Room

L8-04

H6-08

H4-17

A1-65 

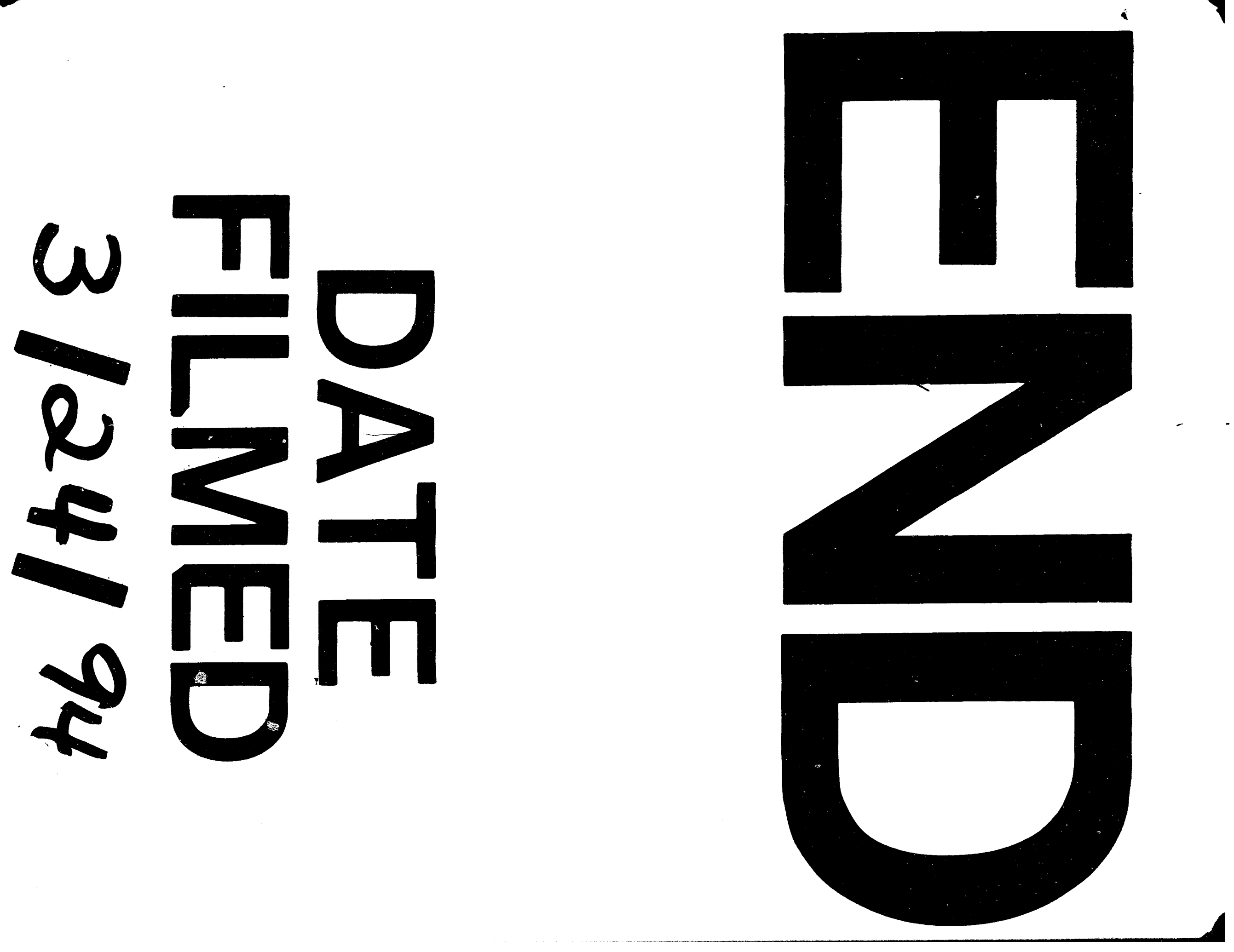

r
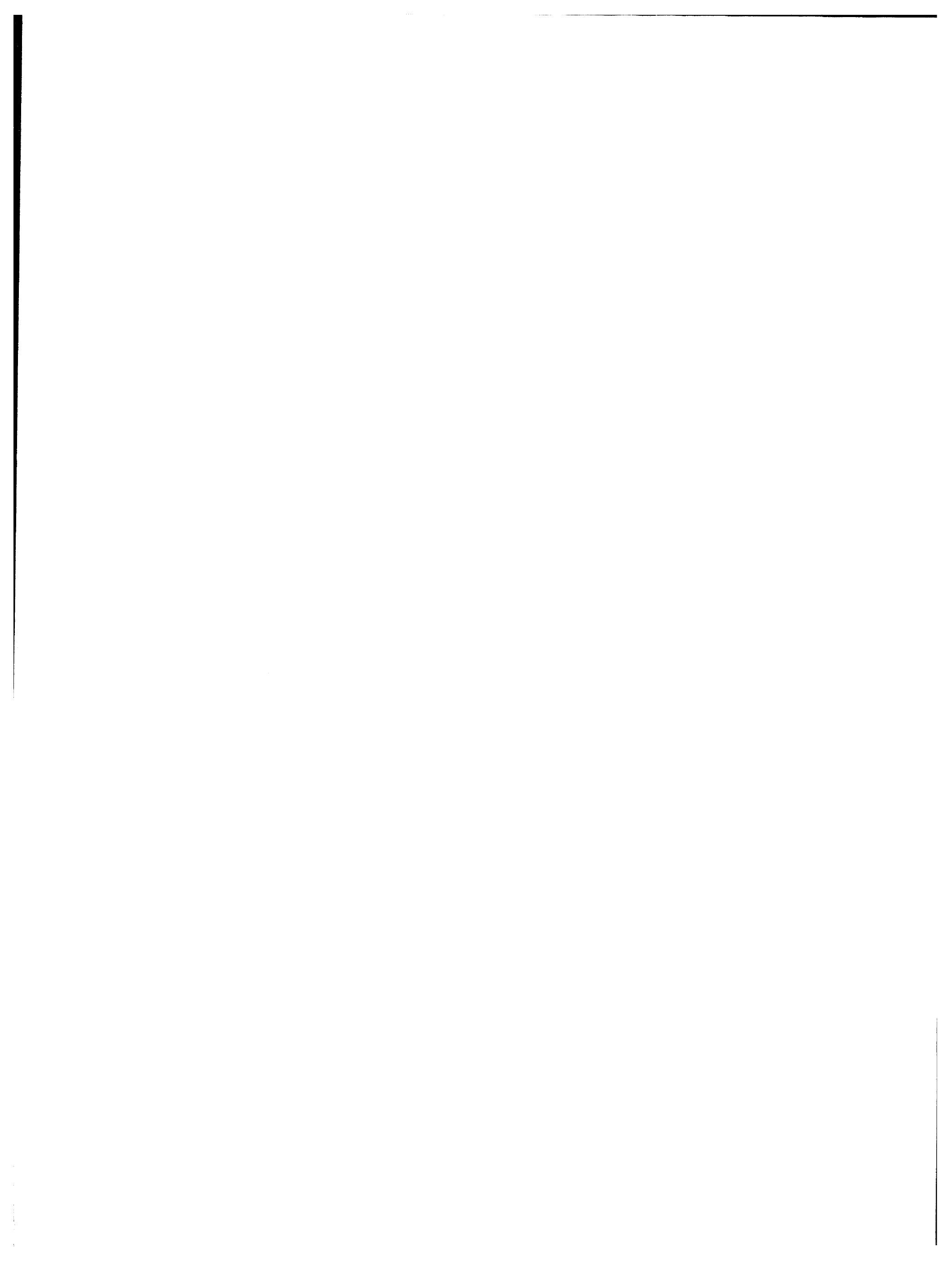